\title{
Уровень радиационной безопасности населения: действующие в стране АЭС и прогнозные оценки для реактора на быстрых нейтронах БРЕСТ в условиях нормальной эксплуатации
}

\author{
Иванов В.К. ${ }^{1,2}$, Чекин С.Ю., ${ }^{1,2}$, Меняйло А.Н., ${ }^{1,2}$, Ловачёв С.С. ${ }^{1,2}$, Корело А.М. ${ }^{1,2}$ \\ ${ }^{1}$ МРНЦ им. А.Ф. Цыба - филиал «НМИЦ радиологии» Минздрава России, Обнинск; \\ 2 ООО «НПК «Мединфо», Обнинск
}

\begin{abstract}
В международных основных нормах безопасности МАГАТЭ (Требование 29) подчёркивается необходимость установления для населения граничных значений не только по величине полученной дозы облучения, но и по значению радиационного риска индукции онкологических заболеваний. Это требование стало выполнимо в связи с завершением крупномасштабных эпидемиологических исследований, прежде всего, в Хиросиме и Нагасаки после атомной бомбардировки этих городов в 1945 г. В действующих в РФ Нормах радиационной безопасности (НРБ-99/2009) утверждены три основных ограничения по величине радиационного риска населения: $5 \cdot 10^{-5}$ год $^{-1}-$ условия нормальной эксплуатации; $1 \cdot 10^{-5}$ год $^{-1}-$ источники потенциального облучения; $1 \cdot 10^{-6}$ год $^{-1}$ - уровень пренебрежимо малого риска. В терминах величины пожизненного атрибутивного риска (LAR) на основе модели МКРЗ (Публикация 103) проведена оценка уровней радиационной безопасности населения, проживающего вблизи действующих в стране АЭС. Технология оценки величины LAR применена для прогнозных оценок уровней безопасности реактора БРЕСТ. Установлено, что значения пожизненного атрибутивного риска онкозаболеваний среди населения, проживающего в регионах размещения действующих в стране АЭС, а также прогнозные оценки для реактора БРЕСТ соответствуют текущим требованиям НРБ-99/2009.
\end{abstract}

Ключевые слова: доза облучения; радиационный риск; требования МАГАТЭ; модель «доза-эфрфект»; эпидемиологические данные Хиросимы и Нагасаки; требования НРБ-99/2009; пожизненный атрибутивный риск; население, проживающее вблизи АЭС; реактор БРЕСТ; пренебрежимо малый риск.

В 2015 г. МАГАТЭ после всестороннего обсуждения в Научном комитете ООН по действию атомной радиации (НКДАР ООН) и Международной комиссии по радиологической защите (МКР3) утвердила свой базовый документ в области радиационной защиты - Международные основные нормы безопасности (МОНБ) [1]. В этом документе впервые подчёркивается (Требование 29), что «Правительство или регулирующий орган устанавливают или утверждают граничные значения дозы и риска, используемые при оптимизации защиты и безопасности лиц из населения». Введение граничных значений не только по дозе облучения, но и по величине радиационного риска возможной индукции онкологических заболеваний обусловлено современной разработкой МКРЗ (Публикация 103) математических моделей «доза-эффект» по итогам крупномасштабных радиационно-эпидемиологических исследований в Хиросиме и Нагасаки после атомной бомбардировки в 1945 г. жителей этих японских городов [2].

В настоящее время при разработке концепции дальнейшего развития ядерной энергетики в России акцент сделан на создании новых технологий, включающих замыкание топливного цикла с использованием реакторов на быстрых нейтронах [3, 4].

Важное значение в практической реализации этой технологии отводится инициированному Госкорпорацией «Росатом» крупномасштабному проекту «Прорыв». В свою очередь, в рам-

Иванов В.К. - зам. директора по научн. работе, Председатель РНКРЗ, чл.-корр. РАН; Чекин С.Ю.* - зав. лаб.; Меняйло А.Н. - вед. научн. сотр., к.б.н.; Ловачёв С.С. - мл. научн. сотр.; Корело А.М. - ст. научн. сотр. МРНЦ им. А.Ф. Цыба - филиал ФГБУ «НМИЦ радиологии» Минздрава России, ООО «НПК «Мединфо».

*Контакты: 249036, Калужская обл., Обнинск, ул. Королёва, 4. Тел.: (484) 399-30-79; e-mail: nrer@obninsk.com. 
ках проекта «Прорыв» необходимо решить ряд сложных проблем современной радиоэкологии, включая обеспечение радиологической защиты населения в терминах конкретных количественных оценок с использованием современных международных и национальных стандартов, ограничивающих дозы облучения и радиационные риски на индивидуальном уровне [5].

В настоящей работе даётся комплексная оценка степени приемлемости радиационных рисков для населения при эксплуатации действующих в стране АЭС и потенциальной эксплуатации опытного демонстрационного энергокомплекса, включающего быстрый реактор БРЕСТОД-300. Учитывая, что работы по опытному демонстрационному энергокомплексу в настоящее время находятся на стадии проектирования, крайне важно получить интегральные оценки уровней радиационной безопасности для населения с целью возможной корректировки проектных решений.

\section{Материалы и методы}

\section{Радиационные риски: международные и национальные стандарты}

В 1954 г. в Японии (Хиросима и Нагасаки) был создан регистр «хибакуси» (лиц, переживших атомную бомбардировку этих городов в 1945 г.). Функционирование регистра продолжается более 60 лет, его основная цель - восстановление реальной зависимости «доза-эффект» с точки зрения возможной радиационно-обусловленной индукции онкологических и неонкологических заболеваний. В частности, было показано, что частота онкозаболеваний солидными раками среди «хибакуси» возросла на 10,7\% [6, 7]. Вместе с тем (рис. 1, 2), в области малых и средних доз облучения (до 150 мЗв) радиационные риски в японской когорте статистически не значимы, что так же подтверждается данными об участниках ликвидации последствий аварии на Чернобыльской АЭС [8].

В настоящее время ограничения радиационного воздействия на человека в международных и национальных стандартах формулируются в терминах относительного и абсолютного риска (рис. 3, 4). Так, граничные значения относительного риска в Англии составляют $20 \%$, в Японии - 10\%, в США - 50\% (с учётом фрактора неопределённости). В действующих в России Нормах радиационной безопасности [9] так же введены граничные значения абсолютного риска для населения.
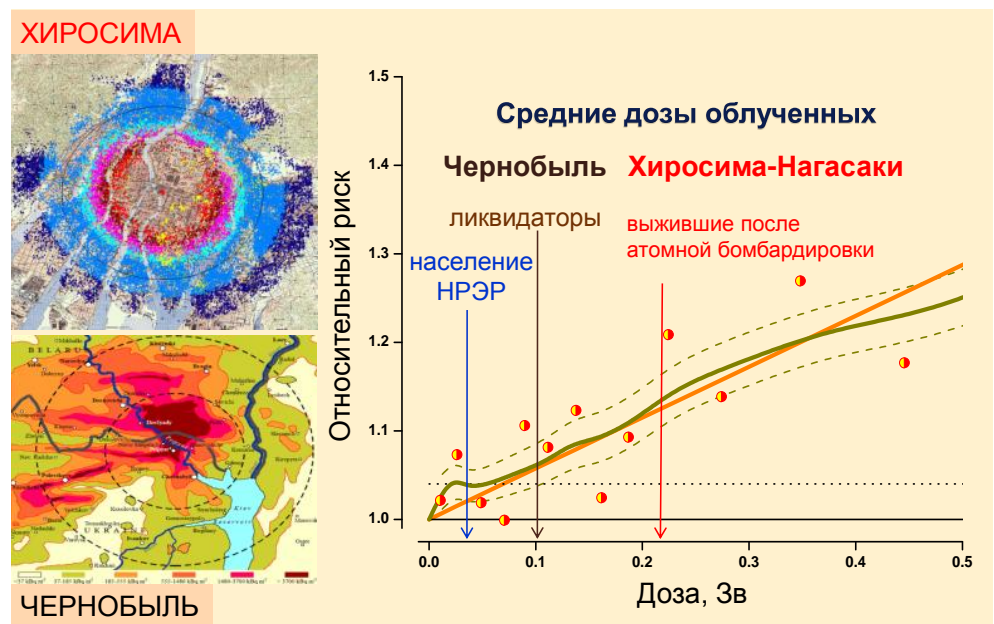

Рис. 1. Риск онкозаболеваемости при малых дозах. 


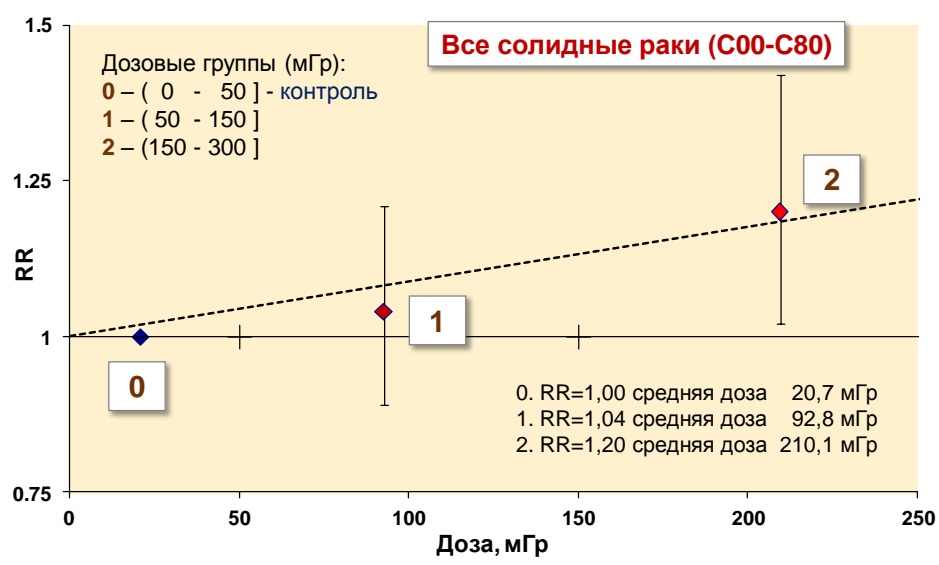

Рис. 2. Дозовая зависимость риска смертности ликвидаторов от онкологической заболеваемости.

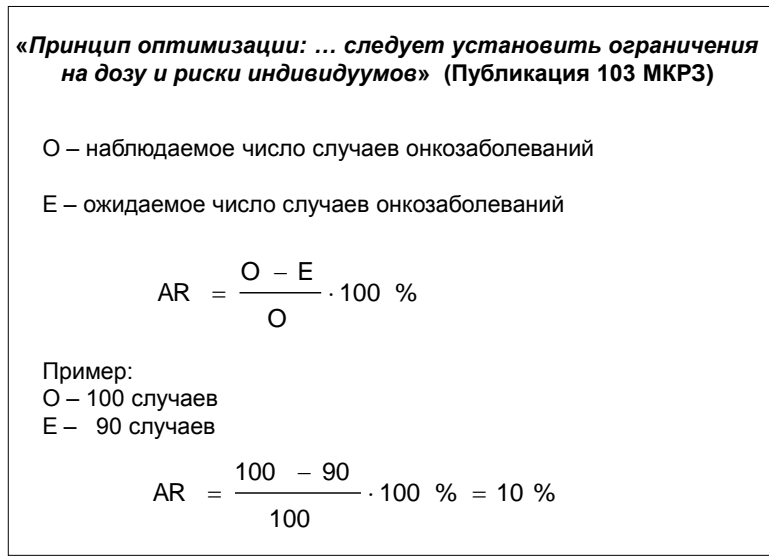

Рис. 3. Избыточный относительный риск.

$$
\begin{aligned}
& \text { В когорте } 100 \text { тыс. человек: } \\
& \text { O - наблюдаемое число случаев онкозаболеваний } \\
& \text { Е - ожидаемое число случаев онкозаболеваний } \\
& \qquad \mathrm{EAR}=\frac{\mathrm{O}-\mathrm{E}}{10^{5}} \\
& \text { Пример: } \\
& \text { O- } 100 \text { случаев } \\
& \text { Е } 90 \text { случаев } \\
& \text { EAR }=\frac{100-90}{10^{5}}=10^{-4}
\end{aligned}
$$

Рис. 4. Избыточный абсолютный риск.

Важно подчеркнуть, что МКРЗ, используя, прежде всего, фрактические данные Хиросимы и Нагасаки, дала современные рекомендации по оценке индивидуального радиационного риска [2], которые были ранее использованы нами для оценки реализуемости принципа радиационной эквивалентности ОЯТ реактора БРЕСТ и природного урана в терминах пожизненного атрибутивного риска LAR (Lifetime Attributable Risk) [5, 10].

Как уже было указано ранее, в соответствии с принятыми недавно Международными основными нормами МАГАТЭ [1] (Требование 29) рекомендуется устанавливать и утверждать граничные значения для населения не только по дозе облучения, но и по величине радиационного риска (рис. 5). Одновременно МКРЗ [2] предлагает использовать для оценки величины радиационного риска не эффрективные, а эквивалентные (органные) дозы от источников внутреннего облучения (рис. 6). Технология оценки радиационных рисков с использованием базы данных тканевых коэффициентов и оценки, в конечном итоге, пожизненного атрибутивного риска LAR показана на рис. 7. Детальная технология оценки величины LAR была приведена нами ранее $[5,11]$. 


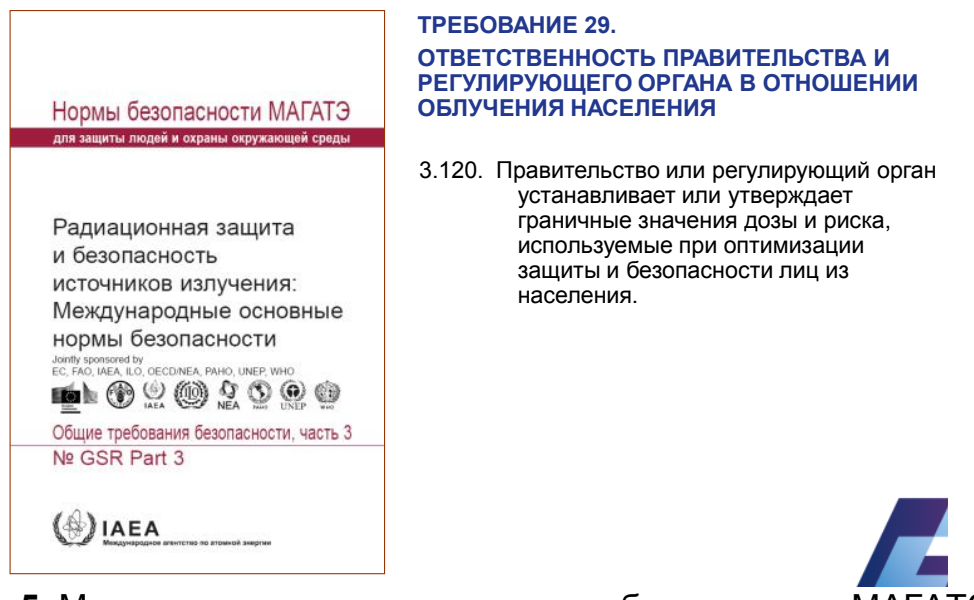

Рис. 5. Международные основные нормы безопасности МАГАТЭ.

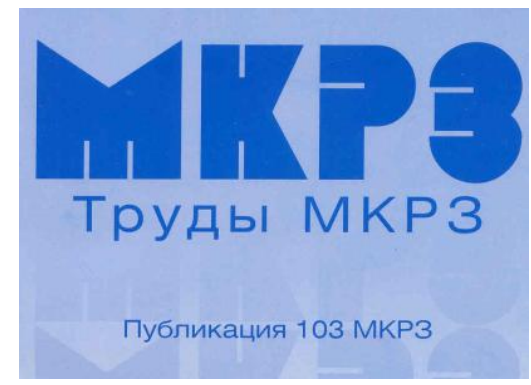

(157) ... Дозы в органах и тканях, а не эффрективные дозы, требуются для оценки вероятности индукции рака у облученных индивидуумов.

Рис. 6. Публикация 103 МКРЗ.

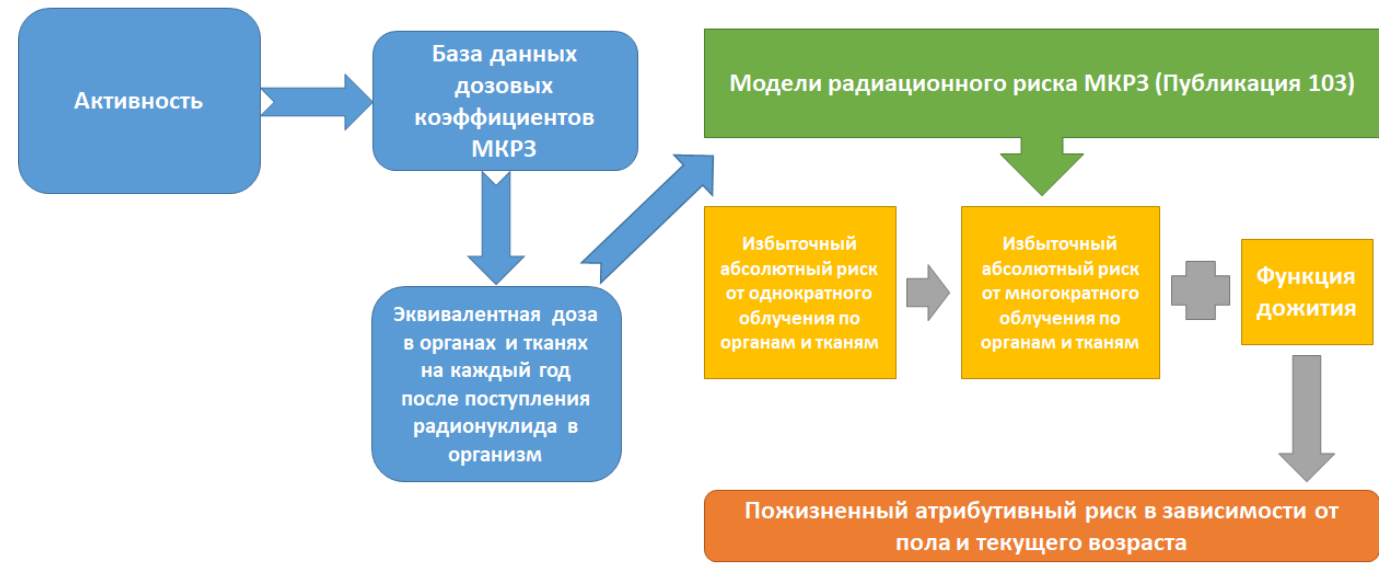

Рис. 7. Технология оценки радиационных рисков.

Как известно, в действующих в стране Нормах радиационной безопасности (НРБ-99/2009) приводятся три ограничения по радиационному риску для населения [9]:

- $5 \cdot 10^{-5}$ год $^{-1}-$ условия нормальной эксплуатации;

- $1 \cdot 10^{-5}$ год $^{-1}-$ источники потенциального облучения;

- $1 \cdot 10^{-6}$ год $^{-1}-$ уровень пренебрежимо малого риска. 
Рассмотрим степень выполнения указанных ограничений на действующих в стране АЭС и в прогнозных оценках проектируемого реактора на быстрых нейтронах БРЕСТ.

\section{Результаты и обсуждение}

В Приложении к настоящей статье (табл. 1-22) приводятся расчётные данные по оценке пожизненного атрибутивного риска (LAR) онкологических заболеваний для населения, проживающего вблизи действующих в стране АЭС. Оценки получены с учётом моделей МКРЗ отдельно для мужского и женского населения в трёх возрастных группах при поступлении радионуклидов (5 лет, 20 лет, 50 лет). Величина LAR также получена как прогнозная оценка для реактора БРЕСТ. Ожидаемые дозы для населения при эксплуатации реактора БРЕСТ оценены в работе [12].

Какой можно сделать основной вывод о впервые проведённой комплексной оценке уровней радиационной безопасности для населения?

Суммарное значение LAR, включающее радиационный риск от ингаляционного и перорального поступления радионуклидов, ниже величины $10^{-6}$, которая в действующих Нормах радиационной безопасности (НРБ-99/2009) отнесена к «пренебрежимо малому риску». Этот основной вывод сохраняется и с учётом потенциального повышения величины риска за счёт дозы внешнего облучения, поскольку доза внешнего облучения, как правило, составляет 20-30\% от суммарной [13].

Следует подчеркнуть, что в современных моделях радиационного риска МКРЗ учтены данные Национального радиационно-эпидемиологического регистра, созданного в России после аварии на Чернобыльской АЭС [14-17].

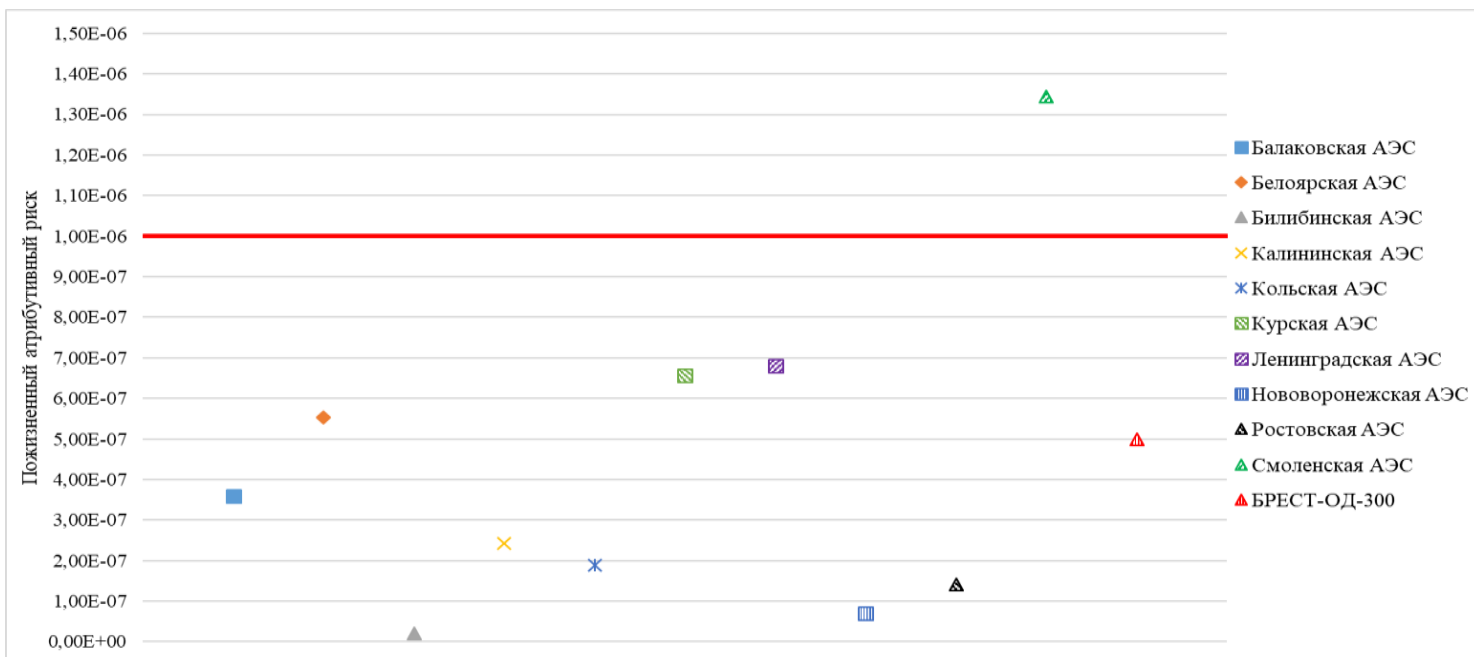

Рис. 8. Пожизненные атрибутивные риски (LAR) онкозаболеваемости после ингаляционного и перорального поступления в организм с питьевой водой и пищей радионуклидов от выбросов и сбросов соответствующих АЭС в течение года (возраст при поступлении - 5 лет; пол - женский).

На рис. 8 приведены значения величины пожизненного атрибутивного риска за счёт ингаляционного и перорального поступления радионуклидов для контрольной группы по модели МКР3 (пол - женский, возраст при облучении - 5 лет). Значения рисков показаны для всех 
10 АЭС и в прогнозном плане для реактора БРЕСТ. Как видно, для всех объектов уровень радиационных рисков соответствует текущим требованиям НРБ-99/2009.

\section{Выводы}

1. В терминах величины пожизненного атрибутивного риска (LAR) на основе модели МКР3 (Публикация 103) проведена оценка уровней радиационной безопасности населения, проживающего вблизи действующих в стране АЭС.

2. Технология оценки величины LAR применена для прогнозных оценок уровней безопасности реактора БРЕСТ.

3. Установлено, что значения пожизненного атрибутивного риска онкозаболеваний среди населения, проживающего в регионах размещения действующих в стране АЭС, а также прогнозные оценки для реактора БРЕСТ соответствуют текущим требованиям НРБ-99/2009. 


\section{Приложение}

Таблица 1

Пожизненные атрибутивные риски (LAR) онкозаболеваемости после ингаляционного и перорального поступления с питьевой водой и пищей в течение года в организм суммарного набора радионуклидов и отдельных радионуклидов от выбросов и сбросов Балаковской АЭС за 2016 г. (возраст при поступлении радионуклидов: 5 лет, 20 лет, 50 лет; мужчины)

\begin{tabular}{|c|c|c|c|c|c|c|c|}
\hline $\begin{array}{l}\text { Радио- } \\
\text { нуклид }\end{array}$ & $\begin{array}{c}\text { Среднегодовая } \\
\text { объёмная } \\
\text { активность } \\
\text { радионуклида } \\
\text { в приземном } \\
\text { слое атмо- } \\
\text { сферного воз- } \\
\left.\text { духа (Бк/м }{ }^{3}\right) \text { и } \\
\text { питьевой воде } \\
(\text { Бк/л) } \\
\end{array}$ & $\begin{array}{l}\text { LAR } \\
\text { (5 лет) }\end{array}$ & $\begin{array}{c}\text { Вклад радио- } \\
\text { нуклида в LAR } \\
\text { (5 лет), \% }\end{array}$ & $\begin{array}{c}\text { LAR } \\
\text { (20 лет) }\end{array}$ & $\begin{array}{c}\text { Вклад радио- } \\
\text { нуклида в LAR } \\
\text { (20 лет), \% }\end{array}$ & $\begin{array}{c}\text { LAR } \\
\text { (50 лет) }\end{array}$ & $\begin{array}{c}\text { Вклад радио- } \\
\text { нуклида в LAR } \\
\text { (50 лет), \% }\end{array}$ \\
\hline 1 & 2 & 3 & 4 & 5 & 6 & 7 & 8 \\
\hline \multicolumn{8}{|c|}{ Ингаляционное поступление } \\
\hline${ }^{137} \mathrm{Cs}$ & 5,23E-07 & $5,71 \mathrm{E}-12$ & 0,72 & $8,28 \mathrm{E}-12$ & 0,89 & $6,57 \mathrm{E}-12$ & 0,90 \\
\hline${ }^{134} \mathrm{Cs}$ & $5,00 \mathrm{E}-07$ & $3,55 \mathrm{E}-12$ & 0,45 & $4,28 \mathrm{E}-12$ & 0,46 & $3,55 \mathrm{E}-12$ & 0,49 \\
\hline${ }^{60} \mathrm{Co}$ & $7,40 \mathrm{E}-05$ & $7,86 \mathrm{E}-10$ & 98,87 & $9,15 \mathrm{E}-10$ & 98,60 & $7,16 \mathrm{E}-10$ & 98,62 \\
\hline LAR & & $7,95 \mathrm{E}-10$ & & $9,28 \mathrm{E}-10$ & & $7,26 \mathrm{E}-10$ & \\
\hline \multicolumn{8}{|c|}{ " Пероральное поступление с питьевой водой } \\
\hline${ }^{173 / C s}$ & 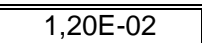 & 4,73E-09 & 5,33 & 6,30E-09 & 12,47 & $2,98 \mathrm{E}-09$ & 12,62 \\
\hline${ }^{60} \mathrm{Co}$ & $1,40 \mathrm{E}-02$ & $8,85 \mathrm{E}-09$ & 9,98 & $1,72 \mathrm{E}-09$ & 3,40 & $7,29 \mathrm{E}-10$ & 3,09 \\
\hline${ }^{3} \mathrm{H}$ & $2,50 \mathrm{E}+01$ & 7,51E-08 & 84,69 & $4,25 \mathrm{E}-08$ & 84,13 & $1,99 \mathrm{E}-08$ & 84,29 \\
\hline LAR & & 8,87E-08 & & $5,05 \mathrm{E}-08$ & & 2,36E-08 & \\
\hline \multicolumn{8}{|c|}{ Пероральное поступление с пищей } \\
\hline${ }^{13} \mathrm{Cs}$ & $\overline{\left({ }^{*}\right)}$ & 9,66E-08 & 100 & 1,13E-07 & 100 & 5,34E-08 & 100 \\
\hline $\begin{array}{c}\text { LAR } \\
\boldsymbol{\Sigma} \text { LAR }\end{array}$ & & $\begin{array}{l}\text { 9,66E-08 } \\
\mathbf{1 , 8 6 E - 0 7}\end{array}$ & & $\begin{array}{l}1,13 \mathrm{E}-07 \\
\mathbf{1 , 6 4 E - 0 7}\end{array}$ & & $\begin{array}{l}5,34 \mathrm{E}-08 \\
7,78 \mathrm{E}-08\end{array}$ & \\
\hline
\end{tabular}

$\left({ }^{*}\right)$ Удельная активность ${ }^{137} \mathrm{Cs}$ в пищевых продуктах (Бк/кг): зерновые - 0,35, овощи - 0,35, рыба - 0,7, мясо - 0,7, молоко $-0,05$.

Таблица 2

Пожизненные атрибутивные риски (LAR) онкозаболеваемости после ингаляционного и перорального поступления с питьевой водой и пищей в течение года в организм суммарного набора радионуклидов и отдельных радионуклидов от выбросов и сбросов Балаковской АЭС за 2016 г. (возраст при поступлении радионуклидов: 5 лет, 20 лет, 50 лет; женщины)

\begin{tabular}{|c|c|c|c|c|c|c|c|}
\hline 1 & 2 & 3 & 4 & 5 & 6 & 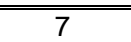 & 8 \\
\hline \multicolumn{8}{|c|}{ Ингаляционное поступление } \\
\hline${ }^{13 /} \mathrm{Cs}$ & 5,23E-07 & $1,1,22 \mathrm{E}-11$ & 0,60 & $1,1,63 \mathrm{E}-11$ & 0,83 & $1,1,25 \mathrm{E}-11$ & 0,93 \\
\hline${ }^{134} \mathrm{Cs}$ & 5.00E-07 & $8,47 \mathrm{E}-12$ & 0,42 & $8,75 \mathrm{E}-12$ & 0,45 & $6.51 \mathrm{E}-12$ & 0,49 \\
\hline${ }^{60} \mathrm{Co}$ & $7,40 \mathrm{E}-05$ & $2,00 \mathrm{E}-09$ & 98,98 & 1,93E-09 & 98,72 & $1,32 \mathrm{E}-09$ & 98,58 \\
\hline LAR & & $2,02 E-09$ & & $1,96 \mathrm{E}-09$ & & $1,34 \mathrm{E}-09$ & \\
\hline \multicolumn{8}{|c|}{ 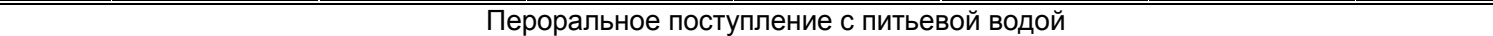 } \\
\hline${ }^{13 / \mathrm{Cs}}$ & $1,20 \mathrm{E}-02$ & $8,83 \mathrm{E}-09$ & 4,98 & $1,1,10 \mathrm{E}-08$ & 12,09 & $4,25 \mathrm{E}-09$ & 12,39 \\
\hline${ }^{60} \mathrm{Co}$ & $1,40 \mathrm{E}-02$ & $1,46 \mathrm{E}-08$ & 8,23 & 2,25E-09 & 2,47 & $8,53 \mathrm{E}-10$ & 2,49 \\
\hline${ }^{3} \mathrm{H}$ & $2,50 \mathrm{E}+01$ & $1,54 \mathrm{E}-07$ & 86,79 & 7,77E-08 & 85,43 & $2,92 \mathrm{E}-08$ & 85,12 \\
\hline LAR & & $1,77 \mathrm{E}-07$ & & $9,10 \mathrm{E}-08$ & & 3,43E-08 & \\
\hline \multicolumn{8}{|c|}{ Пероральное поступление с пищей } \\
\hline$\overline{{ }^{137} \mathrm{Cs}}$ & 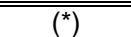 & $\overline{~ 1,80 \mathrm{E}-07}$ & & 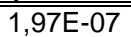 & & $\overline{77,61 \mathrm{E}-08}$ & \\
\hline LAR & & $1,80 \mathrm{E}-07$ & & $1,97 \mathrm{E}-07$ & & $7,61 \mathrm{E}-08$ & \\
\hline$\Sigma$ LAR & & $3,60 \mathrm{E}-07$ & & 2,90E-07 & & $1,12 \mathrm{E}-07$ & \\
\hline
\end{tabular}

(*) Удельная активность ${ }^{137} \mathrm{Cs}$ в пищевых продуктах (Бк/кг): зерновые - 0,35, овощи - 0,35, рыба - 0,7, мясо - 0,7, молоко $-0,05$. 
Таблица 3

Пожизненные атрибутивные риски (LAR) онкозаболеваемости после ингаляционного и перорального поступления с питьевой водой и пищей в течение года в организм суммарного набора радионуклидов и отдельных радионуклидов от выбросов и сбросов Белоярской АЭС за 2016 г. (возраст при поступлении радионуклидов: 5 лет, 20 лет, 50 лет; мужчины)

\begin{tabular}{|c|c|c|c|c|c|c|c|}
\hline $\begin{array}{l}\text { Радио- } \\
\text { нуклид }\end{array}$ & \begin{tabular}{|} 
Среднегодовая \\
объёмная \\
активность \\
радионуклида \\
в приземном \\
слое атмо- \\
сферного воз- \\
духа (Бк/м ${ }^{3}$ ) и \\
питьевой воде \\
(Бк/л) \\
\end{tabular} & $\begin{array}{l}\text { LAR } \\
(5 \text { лет) }\end{array}$ & $\begin{array}{c}\text { Вклад радио- } \\
\text { нуклида в LAR } \\
\text { (5 лет), \% }\end{array}$ & $\begin{array}{c}\text { LAR } \\
\text { (20 лет) }\end{array}$ & $\begin{array}{c}\text { Вклад радио- } \\
\text { нуклида в LAR } \\
\text { (20 лет), \% }\end{array}$ & $\begin{array}{c}\text { LAR } \\
\text { (50 лет) }\end{array}$ & $\begin{array}{c}\text { Вклад радио- } \\
\text { нуклида в LAR } \\
\text { (50 лет), \% }\end{array}$ \\
\hline 1 & 2 & 3 & 4 & 5 & 6 & 7 & 8 \\
\hline \multicolumn{8}{|c|}{ Ингаляционное поступление } \\
\hline 13/Cs & $5,80 \mathrm{E}-07$ & $6,50 \mathrm{E}-12$ & (8,64 & $9,46 \mathrm{E}-12$ & 10,10 & $7,79 \mathrm{E}-12$ & 9,90 \\
\hline${ }^{90} \mathrm{Sr}$ & $3,60 \mathrm{E}-07$ & $1,48 \mathrm{E}-11$ & 19,66 & $2,38 \mathrm{E}-11$ & 25,42 & $2,00 \mathrm{E}-11$ & 25,43 \\
\hline${ }^{134} \mathrm{Cs}$ & $5,00 \mathrm{E}-06$ & $3,65 \mathrm{E}-11$ & 48,49 & $4,39 \mathrm{E}-11$ & 46,89 & $3,77 \mathrm{E}-11$ & 47,93 \\
\hline${ }^{60} \mathrm{Co}$ & 1,10E-06 & $1,20 \mathrm{E}-11$ & 15,94 & $1,39 \mathrm{E}-11$ & 14,85 & $1,13 \mathrm{E}-11$ & 14,37 \\
\hline${ }^{54} \mathrm{Mn}$ & $3,60 \mathrm{E}-06$ & $5,23 \mathrm{E}-12$ & 6,95 & $2,38 \mathrm{E}-12$ & 2,54 & $1,73 \mathrm{E}-12$ & 2,20 \\
\hline${ }^{51} \mathrm{Cr}$ & $1,12 \mathrm{E}-05$ & $2,39 \mathrm{E}-13$ & 0,32 & $1,79 \mathrm{E}-13$ & 0,19 & $1,38 \mathrm{E}-13$ & 0,18 \\
\hline LAR & & $7,53 \mathrm{E}-11$ & & $9,36 \mathrm{E}-11$ & & $7,87 \mathrm{E}-11$ & \\
\hline \multicolumn{8}{|c|}{ Пероральное поступление с питьевой водой } \\
\hline${ }^{13 /} \mathrm{Cs}$ & $2,00 \mathrm{E}-02$ & 7,99E-09 & 17,92 & $1,05 \mathrm{E}-08$ & 33,87 & $5,05 \mathrm{E}-09$ & 34,14 \\
\hline${ }^{3} \mathrm{H}$ & $1,20 \mathrm{E}+01$ & 3,66E-08 & 82,08 & 2,05E-08 & 66,13 & 9,74E-09 & 65,86 \\
\hline LAR & & 4,46E-08 & & $3,10 \mathrm{E}-08$ & & $1,48 \mathrm{E}-08$ & \\
\hline \multicolumn{8}{|c|}{ Пероральное поступление с пищей } \\
\hline${ }^{137} \mathrm{Cs}$ & $\left({ }^{*}\right)$ & 2,33E-07 & 100 & 3,54E-07 & 100 & $1,69 \mathrm{E}-07$ & 100 \\
\hline $\begin{array}{c}\text { LAR } \\
\Sigma \text { LAR }\end{array}$ & & $\begin{array}{l}2,33 \mathrm{E}-07 \\
\mathbf{2 , 7 7 E - 0 7}\end{array}$ & & $\begin{array}{l}3,54 \mathrm{E}-07 \\
3,85 \mathrm{E}-07\end{array}$ & & $\begin{array}{l}\text { 1,69E-07 } \\
\mathbf{1 , 8 4 E - 0 7}\end{array}$ & \\
\hline
\end{tabular}

$\left({ }^{*}\right)$ Удельная активность ${ }^{137} \mathrm{Cs}$ в пищевых продуктах (Бк/кг): пшеница - 2, овощи - 0,18, корнеплоды - 0,25, молоко 0,26 , ягоды -0,14, грибы-1,82, рыба - 1,23.

Таблица 4 Пожизненные атрибутивные риски (LAR) онкозаболеваемости после ингаляционного и перорального поступления с питьевой водой и пищей в течение года в организм суммарного набора радионуклидов и отдельных радионуклидов от выбросов и сбросов Белоярской АЭС за 2016 г. (возраст при поступлении радионуклидов: 5 лет, 20 лет, 50 лет; женщины)

\begin{tabular}{|c|c|c|c|c|c|c|c|}
\hline 1 & 2 & 3 & 4 & 5 & 6 & 7 & 8 \\
\hline \multicolumn{8}{|c|}{ Ингаляционное поступление } \\
\hline${ }^{13 /} \mathrm{Cs}$ & $5,80 \mathrm{E}-07$ & $1,34 \mathrm{E}-11$ & 8,01 & $1,75 \mathrm{E}-11$ & 9,83 & $1,38 \mathrm{E}-11$ & 10,10 \\
\hline${ }^{90} \mathrm{Sr}$ & $3,60 \mathrm{E}-07$ & $2,77 \mathrm{E}-11$ & 16,55 & $4,24 \mathrm{E}-11$ & 23,81 & $3,57 \mathrm{E}-11$ & 26,12 \\
\hline${ }^{134} \mathrm{Cs}$ & $5,00 \mathrm{E}-06$ & $8,54 \mathrm{E}-11$ & 51,03 & $8,51 \mathrm{E}-11$ & 47,80 & $6,47 \mathrm{E}-11$ & 47,33 \\
\hline${ }^{60} \mathrm{Co}$ & $1,10 \mathrm{E}-06$ & $3,03 E-11$ & 18,11 & $2,81 \mathrm{E}-11$ & 15,78 & $1,95 \mathrm{E}-11$ & 14,27 \\
\hline${ }^{54} \mathrm{Mn}$ & $3,60 \mathrm{E}-06$ & $1,01 \mathrm{E}-11$ & 6,04 & $4,63 \mathrm{E}-12$ & 2,60 & $2,78 \mathrm{E}-12$ & 2,03 \\
\hline${ }^{51} \mathrm{Cr}$ & $1,12 \mathrm{E}-05$ & $4,50 \mathrm{E}-13$ & 0,27 & $3,16 \mathrm{E}-13$ & 0,18 & $2,18 \mathrm{E}-13$ & 0,16 \\
\hline LAR & & $1,67 \mathrm{E}-10$ & & $1,78 \mathrm{E}-10$ & & $1,37 \mathrm{E}-10$ & \\
\hline \multicolumn{8}{|c|}{ Пероральное поступление с питьевой водой } \\
\hline${ }^{13 /} \mathrm{Cs}$ & $2,00 \mathrm{E}-02$ & $1,58 \mathrm{E}-08$ & 16,74 & $1,88 \mathrm{E}-08$ & 32,92 & 7,22E-09 & 33,55 \\
\hline${ }^{3} \mathrm{H}$ & $1,20 \mathrm{E}+01$ & $7,86 \mathrm{E}-08$ & 83,26 & $3,83 E-08$ & 67,08 & $1,43 E-08$ & 66,45 \\
\hline LAR & & 9,44E-08 & & 5,71E-08 & & 2,15E-08 & \\
\hline \multicolumn{8}{|c|}{ "Пероральное поступление с пищей } \\
\hline${ }^{137} \mathrm{Cs}$ & $\left({ }^{*}\right)$ & 4,59E-07 & 100 & 6,31E-07 & 100 & $2,42 \mathrm{E}-07$ & 100 \\
\hline LAR & & 4,59E-07 & & $6,31 \mathrm{E}-07$ & & 2,42E-07 & \\
\hline$\Sigma$ LAR & & 5,53E-07 & & 6,88E-07 & & 2,64E-07 & \\
\hline
\end{tabular}

$\left({ }^{*}\right)$ Удельная активность ${ }^{137} \mathrm{Cs}$ в пищевых продуктах (Бк/кг): пшеница - 2, овощи - 0,18, корнеплоды - 0,25, молоко 0,26 , ягоды - 0,14, грибы-1,82, рыба - 1,23. 
Таблица 5

Пожизненные атрибутивные риски (LAR) онкозаболеваемости после ингаляционного и перорального поступления с питьевой водой и пищей в течение года в организм суммарного набора радионуклидов и отдельных радионуклидов от выбросов и сбросов Билибинской АЭС за 2016 г. (возраст при поступлении радионуклидов: 5 лет, 20 лет, 50 лет; мужчины)

\begin{tabular}{|c|c|c|c|c|c|c|c|}
\hline $\begin{array}{l}\text { Радио- } \\
\text { нуклид }\end{array}$ & $\begin{array}{c}\text { Среднегодовая } \\
\text { объёмная } \\
\text { активность } \\
\text { радионуклида } \\
\text { в приземном } \\
\text { слое атмо- } \\
\text { сферного воз- } \\
\left.\text { духа (Бк/м }{ }^{3}\right) \text { и } \\
\text { питьевой воде } \\
(\text { Бк/л) } \\
\end{array}$ & $\begin{array}{l}\text { LAR } \\
\text { (5 лет) }\end{array}$ & $\begin{array}{c}\text { Вклад радио- } \\
\text { нуклида в LAR } \\
\text { (5 лет), \% }\end{array}$ & $\begin{array}{c}\text { LAR } \\
\text { (20 лет) }\end{array}$ & \begin{tabular}{|} 
Вклад радио- \\
нуклида в LAR \\
(20 лет), \%
\end{tabular} & $\begin{array}{c}\text { LAR } \\
\text { (50 лет) }\end{array}$ & $\begin{array}{l}\text { Вклад радио- } \\
\text { нуклида в LAR } \\
\text { (50 лет), \% }\end{array}$ \\
\hline 1 & 2 & 3 & 4 & 5 & 6 & 7 & 8 \\
\hline \multicolumn{8}{|c|}{ Ингаляционное поступление } \\
\hline${ }^{13 /} \mathrm{Cs}$ & $4,4,00 \mathrm{E}-07$ & 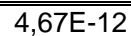 & 0,33 & $6,91 \mathrm{E}-12$ & 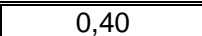 & 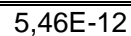 & 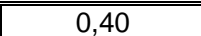 \\
\hline${ }^{134} \mathrm{Cs}$ & $3,00 \mathrm{E}-07$ & $2,23 \mathrm{E}-12$ & 0,16 & $2,78 \mathrm{E}-12$ & 0,16 & $2,33 \mathrm{E}-12$ & 0,17 \\
\hline${ }^{60} \mathrm{Co}$ & $1,28 \mathrm{E}-04$ & $1,41 \mathrm{E}-09$ & 99,47 & $1,70 \mathrm{E}-09$ & 99,42 & $1,35 \mathrm{E}-09$ & 99,41 \\
\hline${ }^{54} \mathrm{Mn}$ & $4,00 \mathrm{E}-07$ & $5,37 \mathrm{E}-13$ & 0,04 & $2,74 \mathrm{E}-13$ & 0,02 & $1,99 \mathrm{E}-13$ & 0,01 \\
\hline${ }^{51} \mathrm{Cr}$ & $7,00 \mathrm{E}-07$ & $1,51 \mathrm{E}-14$ & 0,00 & $1,17 \mathrm{E}-14$ & 0,00 & $8,85 \mathrm{E}-15$ & 0,00 \\
\hline LAR & & $1,42 \mathrm{E}-09$ & & $1,71 \mathrm{E}-09$ & & $1,36 \mathrm{E}-09$ & \\
\hline \multicolumn{8}{|c|}{ Пероральное поступление с питьевой водой } \\
\hline${ }^{13 /} \mathrm{Cs}$ & 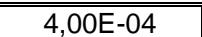 & 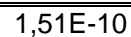 & 32,68 & $2,2,10 \mathrm{E}-10$ & 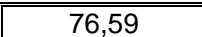 & 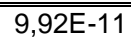 & 79,87 \\
\hline${ }^{60} \mathrm{Co}$ & $5,00 \mathrm{E}-04$ & $3,11 \mathrm{E}-10$ & 67,32 & $6,42 E-11$ & 23,41 & $2,50 \mathrm{E}-11$ & 20,13 \\
\hline LAR & & $4,62 \mathrm{E}-10$ & & $2,74 \mathrm{E}-10$ & & $1,24 \mathrm{E}-10$ & \\
\hline \multicolumn{8}{|c|}{ "Пероральное поступление с пищей } \\
\hline & 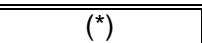 & $\overline{3,71 \mathrm{E}-09}$ & 43,70 & 5,44E-09 & 10,31 & 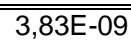 & $\overline{14,71}$ \\
\hline${ }^{137} \mathrm{Cs}$ & $\left({ }^{\star \star}\right)$ & $1,56 \mathrm{E}-09$ & 18,37 & $4,60 \mathrm{E}-08$ & 87,19 & $2,17 \mathrm{E}-08$ & 83,32 \\
\hline${ }^{60} \mathrm{Co}$ & $\left({ }^{* \star \star}\right)$ & $3,22 \mathrm{E}-09$ & 37,93 & $1,32 \mathrm{E}-09$ & 2,50 & $5,13 \mathrm{E}-10$ & 1,97 \\
\hline LAR & & 8,49E-09 & & $5,28 \mathrm{E}-08$ & & 2,60E-08 & \\
\hline$\Sigma$ LAR & & $1,04 \mathrm{E}-08$ & & $5,48 \mathrm{E}-08$ & & 2,76E-08 & \\
\hline
\end{tabular}

(*) Удельная активность ${ }^{90} \mathrm{Sr}$ в пищевых продуктах (Бк/кг): ягоды - 0,21, грибы - 0,27; $\left({ }^{* *}\right)$ Удельная активность ${ }^{137} \mathrm{Cs}$ в пищевых продуктах (Бк/кг): ягоды - 0,4, грибы - 6,2; $\left({ }^{* * *}\right)$ Удельная активность ${ }^{60}$ Со в пищевых продуктах (Бк/кг): ягоды - 0,5, грибы - 0,5.

Таблица 6

Пожизненные атрибутивные риски (LAR) онкозаболеваемости после ингаляционного и перорального поступления с питьевой водой и пищей в течение года в организм суммарного набора радионуклидов и отдельных радионуклидов от выбросов и сбросов Билибинской АЭС за 2016 г. (возраст при поступлении радионуклидов: 5 лет, 20 лет, 50 лет; женщины)

\begin{tabular}{|c|c|c|c|c|c|c|c|}
\hline 1 & 2 & 3 & 4 & 5 & 6 & 7 & 8 \\
\hline \multicolumn{8}{|c|}{ Ингаляционное поступление } \\
\hline${ }^{13 /} \mathrm{Cs}$ & $4,00 \mathrm{E}-07$ & $2,26 \mathrm{E}-11$ & 0,36 & $3,3,76 \mathrm{E}-11$ & 0,47 & $4,82 \mathrm{E}-11$ & 0,49 \\
\hline${ }^{134} \mathrm{Cs}$ & $3,00 \mathrm{E}-07$ & $1,02 E-11$ & 0,16 & $1,37 \mathrm{E}-11$ & 0,17 & $1,75 \mathrm{E}-11$ & 0,18 \\
\hline${ }^{60} \mathrm{Co}$ & $1,28 \mathrm{E}-04$ & $6,30 \mathrm{E}-09$ & 99,46 & 7,99E-09 & 99,35 & 9,73E-09 & 99,32 \\
\hline${ }^{54} \mathrm{Mn}$ & $4,00 \mathrm{E}-07$ & $1,58 \mathrm{E}-12$ & 0,02 & $1,03 \mathrm{E}-12$ & 0,01 & $1,11 \mathrm{E}-12$ & 0,01 \\
\hline${ }^{b 1} \mathrm{Cr}$ & $7,00 \mathrm{E}-07$ & $4,84 \mathrm{E}-14$ & 0,00 & $4,52 \mathrm{E}-14$ & 0,00 & $5,35 \mathrm{E}-14$ & 0,00 \\
\hline LAR & & $6,33 \mathrm{E}-09$ & & 8,04E-09 & & 9,80E-09 & \\
\hline \multicolumn{8}{|c|}{ Пероральное поступление с питьевой водой } \\
\hline${ }^{13 /} \mathrm{Cs}$ & $4,00 \mathrm{E}-04$ & $3,71 \mathrm{E}-10$ & 36,23 & $4,99 \mathrm{E}-10$ & 82,07 & $3,11 \mathrm{E}-10$ & 83,18 \\
\hline${ }^{60} \mathrm{Co}$ & $5,00 \mathrm{E}-04$ & $6,53 \mathrm{E}-10$ & 63,77 & $1,09 \mathrm{E}-10$ & 17,93 & $6,29 E-11$ & 16,82 \\
\hline LAR & & $1,02 E-09$ & & $6,08 \mathrm{E}-10$ & & $3,74 \mathrm{E}-10$ & \\
\hline \multicolumn{8}{|c|}{ Пероральное поступление с пищей } \\
\hline${ }^{90} \mathrm{Sr}$ & $\left({ }^{*}\right)$ & $2,44 \mathrm{E}-09$ & 18,73 & 4,09E-09 & 3,55 & 3,57E-09 & 4,89 \\
\hline${ }^{13 /} \mathrm{Cs}$ & $(* *)$ & 3,84E-09 & 29,47 & $1,09 \mathrm{E}-07$ & 94,52 & $6,81 \mathrm{E}-08$ & 93,34 \\
\hline${ }^{60} \mathrm{Co}$ & $\left({ }^{* * *}\right)$ & $6,75 \mathrm{E}-09$ & 51,80 & $2,23 \mathrm{E}-09$ & 1,93 & 1,29E-09 & 1,77 \\
\hline LAR & & $1,30 \mathrm{E}-08$ & & $1,15 \mathrm{E}-07$ & & 7,30E-08 & \\
\hline$\Sigma$ LAR & & 2,04E-08 & & 1,24E-07 & & 8,31E-08 & \\
\hline
\end{tabular}

(*) Удельная активность ${ }^{90} \mathrm{Sr}$ в пищевых продуктах (Бк/кг): ягоды - 0,21, грибы - 0,27; $\left({ }^{* *}\right)$ Удельная активность ${ }^{137} \mathrm{Cs}$ в пищевых продуктах (Бк/кг): ягоды - 0,4, грибы - 6,2; $\left({ }^{* * *}\right)$ Удельная активность ${ }^{60}$ Со в пищевых продуктах (Бк/кг): ягоды - 0,5, грибы - 0,5. 
Таблица 7

Пожизненные атрибутивные риски (LAR) онкозаболеваемости после ингаляционного и перорального поступления с питьевой водой и пищей в течение года в организм суммарного набора радионуклидов и отдельных радионуклидов от выбросов и сбросов Калининской АЭС за 2016 г. (возраст при поступлении радионуклидов: 5 лет, 20 лет, 50 лет; мужчины)

\begin{tabular}{|c|c|c|c|c|c|c|c|}
\hline $\begin{array}{l}\text { Радио- } \\
\text { нуклид }\end{array}$ & $\begin{array}{c}\text { Среднегодовая } \\
\text { объёмная } \\
\text { активность } \\
\text { радионуклида } \\
\text { в приземном } \\
\text { слое атмо- } \\
\text { сферного воз- } \\
\left.\text { духа (Бк/м }{ }^{3}\right) \text { и } \\
\text { питьевой воде } \\
(\text { Бк/л) } \\
\end{array}$ & $\begin{array}{l}\text { LAR } \\
\text { (5 лет) }\end{array}$ & $\begin{array}{c}\text { Вклад радио- } \\
\text { нуклида в LAR } \\
\text { (5 лет), \% }\end{array}$ & $\begin{array}{c}\text { LAR } \\
\text { (20 лет) }\end{array}$ & \begin{tabular}{|c} 
Вклад радио- \\
нуклида в LAR \\
(20 лет), \%
\end{tabular} & $\begin{array}{c}\text { LAR } \\
\text { (50 лет) }\end{array}$ & $\begin{array}{c}\text { Вклад радио- } \\
\text { нуклида в LAR } \\
\text { (50 лет), \% }\end{array}$ \\
\hline 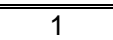 & 2 & 34 & 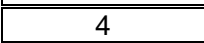 & 5 & 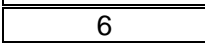 & $\overline{77}$ & 8 \\
\hline \multicolumn{8}{|c|}{ Ингаляционное поступление } \\
\hline${ }^{137} \mathrm{Cs}$ & $3,45 \mathrm{E}-07$ & 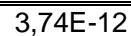 & 40 & $5,40 \mathrm{E}-12$ & $4 \overline{47,94}$ & 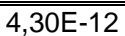 & $\overline{407,82}$ \\
\hline${ }^{1374} \mathrm{Cs}$ & $2,46 \mathrm{E}-07$ & $1,74 \mathrm{E}-12$ & 19,48 & $2,08 \mathrm{E}-12$ & 18,47 & $1,73 \mathrm{E}-12$ & 19,24 \\
\hline${ }^{60} \mathrm{Co}$ & $2,98 \mathrm{E}-07$ & $3,14 \mathrm{E}-12$ & 35,16 & $3,64 \mathrm{E}-12$ & 32,32 & $2,86 \mathrm{E}-12$ & 31,81 \\
\hline${ }^{54} \mathrm{Mn}$ & $2,25 \mathrm{E}-07$ & $3,11 \mathrm{E}-13$ & 3,48 & $1,44 \mathrm{E}-13$ & 1,28 & $1,02 \mathrm{E}-13$ & 1,13 \\
\hline LAR & & $8,93 \mathrm{E}-12$ & & $1,13 \mathrm{E}-11$ & & $8,99 \mathrm{E}-12$ & \\
\hline \multicolumn{8}{|c|}{ Пероральное поступление с питьевой водой } \\
\hline${ }^{137} \mathrm{Cs}$ & $\begin{array}{l}1,90 \mathrm{E}-03 \\
\end{array}$ & $\overline{77,43 \mathrm{E}-10}$ & $\mid 2,21$ & $9,9,86 \mathrm{E}-10$ & 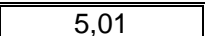 & 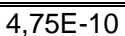 & 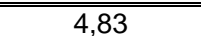 \\
\hline${ }^{3} \mathrm{H}$ & $1,00 \mathrm{E}+01$ & $2,98 \mathrm{E}-08$ & 88,71 & $1,68 \mathrm{E}-08$ & 85,38 & $8,01 \mathrm{E}-09$ & 81,44 \\
\hline${ }^{90} \mathrm{Sr}$ & $1,80 \mathrm{E}-03$ & $3,05 \mathrm{E}-09$ & 9,08 & $1,89 \mathrm{E}-09$ & 9,61 & $1,35 \mathrm{E}-09$ & 13,73 \\
\hline LAR & & $3,36 \mathrm{E}-08$ & & 1,97E-08 & & $9,84 \mathrm{E}-09$ & \\
\hline \multicolumn{8}{|c|}{ "Пероральное поступление с пищей } \\
\hline${ }^{137} \mathrm{Cs}$ & 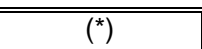 & 3,16E-08 & 14,59 & $3,80 \mathrm{E}-08$ & 22,22 & 1,83E-08 & 16,12 \\
\hline${ }^{90} \mathrm{Sr}$ & $\left({ }^{* *}\right)$ & $1,85 \mathrm{E}-07$ & 85,41 & $1,33 \mathrm{E}-07$ & 77,78 & $9,52 \mathrm{E}-08$ & 83,88 \\
\hline $\begin{array}{c}\text { LAR } \\
\Sigma \text { LAR }\end{array}$ & & $\begin{array}{l}2,17 \mathrm{E}-07 \\
\mathbf{2}, 50 \mathrm{E}-07\end{array}$ & & $\begin{array}{l}1,71 \mathrm{E}-07 \\
\mathbf{1}, 91 \mathrm{E}-07\end{array}$ & & $\begin{array}{l}1,14 \mathrm{E}-07 \\
1,23 \mathrm{E}-07\end{array}$ & \\
\hline
\end{tabular}

$\left({ }^{*}\right)$ Удельная активность ${ }^{137} \mathrm{Cs}$ в пищевых продуктах (Бк/кг): корнеплоды - 0,06, овощи - 0,05, ягоды - 1,7, грибы - 0,65, молоко 0,03, мясо - 0,07, рыба - 0,38; ${ }^{* *}$ ) Удельная активность ${ }^{90} \mathrm{Sr}$ в пищевых продуктах (Бк/кг): корнеплоды - 0,21, овощи - 0,1, ягоды $-0,21$, грибы-0,07, молоко -0,06, мясо - 0,09, рыба - 0,34.

Таблица 8

Пожизненные атрибутивные риски (LAR) онкозаболеваемости после ингаляционного и перорального поступления с питьевой водой и пищей в течение года в организм суммарного набора радионуклидов и отдельных радионуклидов от выбросов и сбросов Калининской АЭС за 2016 г. (возраст при поступлении радионуклидов: 5 лет, 20 лет, 50 лет; женщины)

\begin{tabular}{|c|c|c|c|c|c|c|c|}
\hline 1 & 2 & 34 & 4 & 5 & 6 & 7 & 8 \\
\hline \multicolumn{8}{|c|}{ Ингаляционное поступление } \\
\hline${ }^{13 /} \mathrm{Cs}$ & $3,45 \mathrm{E}-07$ & 7,94E-12 & 37,93 & $1,05 \mathrm{E}-11$ & 46,23 & $7,92 \mathrm{E}-12$ & 48,59 \\
\hline${ }^{13 / 4} \mathrm{Cs}$ & $2,46 \mathrm{E}-07$ & $4,19 \mathrm{E}-12$ & 20,02 & $4,23 \mathrm{E}-12$ & 18,63 & $3,09 \mathrm{E}-12$ & 18,96 \\
\hline${ }^{60} \mathrm{Co}$ & $2,98 \mathrm{E}-07$ & $8,18 \mathrm{E}-12$ & 39,08 & $7,69 \mathrm{E}-12$ & 33,86 & $5,12 \mathrm{E}-12$ & 31,41 \\
\hline${ }^{54} \mathrm{Mn}$ & 2,25E-07 & $6,23 \mathrm{E}-13$ & 2,98 & $2,91 \mathrm{E}-13$ & 1,28 & $1,70 \mathrm{E}-13$ & 1,04 \\
\hline LAR & & $2,09 \mathrm{E}-11$ & & $2,27 \mathrm{E}-11$ & & $1,63 \mathrm{E}-11$ & \\
\hline \multicolumn{8}{|c|}{$\begin{array}{ll}\text { Пероральное поступление с питьевой водой }\end{array}$} \\
\hline${ }^{137} \mathrm{Cs}$ & $1,90 \mathrm{E}-03$ & $\overline{1,48 \mathrm{E}-09}$ & 2,16 & 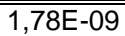 & 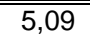 & 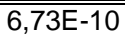 & 4,96 \\
\hline${ }^{3} \mathrm{H}$ & $1.00 \mathrm{E}+01$ & $6,51 \mathrm{E}-08$ & 95,16 & $3,18 \mathrm{E}-08$ & 90,99 & $1,17 \mathrm{E}-08$ & 86,20 \\
\hline${ }^{90} \mathrm{Sr}$ & $1,80 \mathrm{E}-03$ & 1,83E-09 & 2,68 & 1,37E-09 & 3,92 & $1,20 \mathrm{E}-09$ & 8,84 \\
\hline LAR & & $6,84 \mathrm{E}-08$ & & $3,50 \mathrm{E}-08$ & & $1,36 \mathrm{E}-08$ & \\
\hline \multicolumn{8}{|c|}{ Пероральное поступление с пищей } \\
\hline${ }^{137} \mathrm{Cs}$ & ("*) & $6,30 \mathrm{E}-08$ & 36,21 & $26,86 \mathrm{E}-08$ & $4 \quad 41,55$ & $2,59 \mathrm{E}-08$ & 23,40 \\
\hline${ }^{90} \mathrm{Sr}$ & $\left({ }^{\star \star}\right)$ & 1,11E-07 & 63,79 & $9,65 E-08$ & 58,45 & $8,48 \mathrm{E}-08$ & 76,60 \\
\hline $\begin{array}{l}\text { LAR } \\
\Sigma \text { LAR }\end{array}$ & & $\begin{array}{l}1,74 \mathrm{E}-07 \\
2,42 \mathrm{E}-07\end{array}$ & & $\begin{array}{l}1,65 \mathrm{E}-07 \\
200 \mathrm{E}-07\end{array}$ & & $\begin{array}{l}1,11 \mathrm{E}-07 \\
124 \mathrm{E}-07\end{array}$ & \\
\hline
\end{tabular}

(*) Удельная активность ${ }^{137} \mathrm{Cs}$ в пищевых продуктах (Бк/кг): корнеплоды - 0,06, овощи - 0,05, ягоды - 1,7, грибы - 0,65, молоко 0,03 , мясо - 0,07, рыба - 0,38; $\left({ }^{* *}\right)$ Удельная активность ${ }^{90} \mathrm{Sr}$ в пищевых продуктах (Бк/кг): корнеплоды - 0,21, овощи - 0,1, ягоды $-0,21$, грибы-0,07, молоко-0,06, мясо-0,09, рыба-0,34. 
Таблица 9

Пожизненные атрибутивные риски (LAR) онкозаболеваемости после ингаляционного и перорального поступления с питьевой водой и пищей в течение года в организм суммарного набора радионуклидов и отдельных радионуклидов от выбросов и сбросов Кольской АЭС за 2016 г. (возраст при поступлении радионуклидов: 5 лет, 20 лет, 50 лет; мужчины)

\begin{tabular}{|c|c|c|c|c|c|c|c|}
\hline $\begin{array}{l}\text { Радио- } \\
\text { нуклид }\end{array}$ & $\begin{array}{c}\text { Среднегодовая } \\
\text { объёмная } \\
\text { активность } \\
\text { радионуклида } \\
\text { в приземном } \\
\text { слое атмо- } \\
\text { сферного воз- } \\
\text { духа (Бк/м³) и } \\
\text { питьевой воде } \\
\text { (Бк/л) }\end{array}$ & $\begin{array}{c}\text { LAR } \\
\text { (5 лет) }\end{array}$ & $\begin{array}{c}\text { Вклад радио- } \\
\text { нуклида в LAR } \\
\text { (5 лет), \% }\end{array}$ & $\begin{array}{c}\text { LAR } \\
\text { (20 лет) }\end{array}$ & $\begin{array}{c}\text { Вклад радио- } \\
\text { нуклида в LAR } \\
\text { (20 лет), \% }\end{array}$ & $\begin{array}{c}\text { LAR } \\
\text { (50 лет) }\end{array}$ & $\begin{array}{c}\text { Вклад радио- } \\
\text { нуклида в LAR } \\
\text { (50 лет), \% }\end{array}$ \\
\hline 1 & 2 & 3 & 4 & 5 & 6 & 7 & 8 \\
\hline \multicolumn{8}{|c|}{ Ингаляционное поступление } \\
\hline 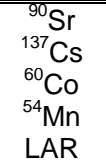 & $\begin{array}{l}3,10 \mathrm{E}-08 \\
1,20 \mathrm{E}-06 \\
1,20 \mathrm{E}-06 \\
1,00 \mathrm{E}-06\end{array}$ & $\begin{array}{l}1,27 \mathrm{E}-12 \\
1,36 \mathrm{E}-11 \\
1,35 \mathrm{E}-11 \\
1,53 \mathrm{E}-12 \\
2,99 \mathrm{E}-11\end{array}$ & \begin{tabular}{|c|}
4,25 \\
45,48 \\
45,15 \\
5,12 \\
\end{tabular} & $\begin{array}{l}2,05 \mathrm{E}-12 \\
1,96 \mathrm{E}-11 \\
1,54 \mathrm{E}-11 \\
6,85 \mathrm{E}-13 \\
3,77 \mathrm{E}-11\end{array}$ & $\begin{array}{c}5,43 \\
51,94 \\
40,81 \\
1,82\end{array}$ & $\begin{array}{l}1,69 \mathrm{E}-12 \\
1,59 \mathrm{E}-11 \\
1,22 \mathrm{E}-11 \\
4,81 \mathrm{E}-13 \\
3,03 \mathrm{E}-11\end{array}$ & $\begin{array}{c}5,58 \\
52,53 \\
40,30 \\
1,59\end{array}$ \\
\hline \multicolumn{8}{|c|}{ " Пероральное поступление с питьевой водой } \\
\hline $\begin{array}{l}{ }^{137} \mathrm{Cs} \\
\text { LAR }\end{array}$ & 1,20E-03 & $\begin{array}{l}5,26 \mathrm{E}-10 \\
5,26 \mathrm{E}-10\end{array}$ & 100 & $\begin{array}{l}6,86 \mathrm{E}-10 \\
6,86 \mathrm{E}-10 \\
\end{array}$ & 100 & $\begin{array}{l}3,27 \mathrm{E}-10 \\
3,27 \mathrm{E}-10\end{array}$ & 100 \\
\hline \multicolumn{8}{|c|}{ Пероральное поступление с пищей } \\
\hline $\begin{array}{c}{ }^{137} \mathrm{Cs} \\
\text { LAR } \\
\Sigma \text { LAR }\end{array}$ & $\overline{\left({ }^{*}\right)}$ & $\begin{array}{l}1,03 E-07 \\
1,03 E-07 \\
\mathbf{1 , 0 4 E - 0 7}\end{array}$ & 100 & $\begin{array}{l}8,07 \mathrm{E}-08 \\
8,07 \mathrm{E}-08 \\
\mathbf{8 , 1 4 E - 0 8}\end{array}$ & 100 & $\begin{array}{l}3,85 \mathrm{E}-08 \\
3,85 \mathrm{E}-08 \\
3,88 \mathrm{E}-08\end{array}$ & 100 \\
\hline
\end{tabular}

(*) Удельная активность ${ }^{137} \mathrm{Cs}$ в пищевых продуктах (Бк/кг): грибы - 3,4, ягоды - 6,3, рыба - 2,5.

Таблица 10

Пожизненные атрибутивные риски (LAR) онкозаболеваемости после ингаляционного и перорального поступления с питьевой водой и пищей в течение года в организм суммарного набора радионуклидов и отдельных радионуклидов от выбросов и сбросов Кольской АЭС за 2016 г. (возраст при поступлении радионуклидов: 5 лет, 20 лет, 50 лет; женщины)

\begin{tabular}{|c|c|c|c|c|c|c|c|}
\hline 1 & 2 & 3 & 4 & 5 & 6 & 7 & 8 \\
\hline \multicolumn{8}{|c|}{ Ингаляционное поступление } \\
\hline $\begin{array}{l}{ }^{90} \mathrm{Sr} \\
{ }^{13 /} \mathrm{Cs} \\
{ }^{60} \mathrm{Co} \\
{ }^{54} \mathrm{Mn} \\
\text { LAR }\end{array}$ & $\begin{array}{l}3,10 \mathrm{E}-08 \\
1,20 \mathrm{E}-06 \\
1,20 \mathrm{E}-06 \\
1,00 \mathrm{E}-06\end{array}$ & $\begin{array}{l}2,52 \mathrm{E}-12 \\
2,89 \mathrm{E}-11 \\
3,38 \mathrm{E}-11 \\
2,83 \mathrm{E}-12 \\
6,81 \mathrm{E}-11\end{array}$ & $\begin{array}{c}3,70 \\
42,47 \\
49,67 \\
4,16\end{array}$ & $\begin{array}{l}3,96 \mathrm{E}-12 \\
3,88 \mathrm{E}-11 \\
3,24 \mathrm{E}-11 \\
1,34 \mathrm{E}-12 \\
7,65 \mathrm{E}-11\end{array}$ & $\begin{array}{c}5,18 \\
50,72 \\
42,35 \\
1,75\end{array}$ & $\begin{array}{l}3,25 \mathrm{E}-12 \\
3,01 \mathrm{E}-11 \\
2,25 \mathrm{E}-11 \\
8,20 \mathrm{E}-13 \\
5,67 \mathrm{E}-11\end{array}$ & $\begin{array}{c}5,73 \\
53,11 \\
39,70 \\
1,45\end{array}$ \\
\hline \multicolumn{8}{|c|}{ Пероральное поступление с питьевой водой } \\
\hline $\begin{array}{l}{ }^{137} \mathrm{Cs} \\
\mathrm{LAR}\end{array}$ & 1,20E-03 & $\begin{array}{l}9,54 \mathrm{E}-10 \\
9,54 \mathrm{E}-10\end{array}$ & 100 & $\begin{array}{l}1,15 \mathrm{E}-09 \\
1,15 \mathrm{E}-09\end{array}$ & 100 & $\begin{array}{l}4,49 \mathrm{E}-10 \\
4,49 \mathrm{E}-10\end{array}$ & 100 \\
\hline \multicolumn{8}{|c|}{ Пероральное поступление с пищей } \\
\hline $\begin{array}{c}{ }^{13 /} \mathrm{Cs} \\
\text { LAR } \\
\Sigma \text { LAR }\end{array}$ & 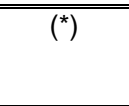 & $\begin{array}{l}1,87 \mathrm{E}-07 \\
\text { 1,87E-07 } \\
\mathbf{1 , 8 8 E - 0 7}\end{array}$ & 100 & $\begin{array}{l}\text { 1,36E-07 } \\
\text { 1,36E-07 } \\
\mathbf{1 , 3 7 E - 0 7}\end{array}$ & 100 & $\begin{array}{l}\text { 5,28E-08 } \\
\text { 5,28E-08 } \\
\text { 5,34E-08 }\end{array}$ & 100 \\
\hline
\end{tabular}

(*) Удельная активность ${ }^{137} \mathrm{Cs}$ в пищевых продуктах (Бк/кг): грибы - 3,4, ягоды - 6,3, рыба - 2,5. 
Таблица 11

Пожизненные атрибутивные риски (LAR) онкозаболеваемости после ингаляционного и перорального поступления с питьевой водой и пищей в течение года в организм суммарного набора радионуклидов и отдельных радионуклидов от выбросов и сбросов Курской АЭС за 2016 г. (возраст при поступлении радионуклидов: 5 лет, 20 лет, 50 лет; мужчины)

\begin{tabular}{|c|c|c|c|c|c|c|c|}
\hline $\begin{array}{l}\text { Радио- } \\
\text { нуклид }\end{array}$ & $\begin{array}{c}\text { Среднегодовая } \\
\text { объёмная } \\
\text { активность } \\
\text { радионуклида } \\
\text { в приземном } \\
\text { слое атмо- } \\
\text { сферного воз- } \\
\text { духа (Бк/м³) и } \\
\text { питьевой воде } \\
\text { (Бк/л) }\end{array}$ & $\begin{array}{c}\text { LAR } \\
\text { (5 лет) }\end{array}$ & $\begin{array}{c}\text { Вклад радио- } \\
\text { нуклида в LAR } \\
\text { (5 лет), \% }\end{array}$ & $\begin{array}{c}\text { LAR } \\
\text { (20 лет) }\end{array}$ & $\begin{array}{c}\text { Вклад радио- } \\
\text { нуклида в LAR } \\
\text { (20 лет), \% }\end{array}$ & $\begin{array}{c}\text { LAR } \\
(50 \text { лет) }\end{array}$ & $\begin{array}{c}\text { Вклад радио- } \\
\text { нуклида в LAR } \\
\text { (50 лет), \% }\end{array}$ \\
\hline \multicolumn{8}{|c|}{ Ингаляционное поступление } \\
\hline${ }^{24} \mathrm{Na}$ & 1,00E-07 & $1,36 \mathrm{E}-13$ & 0,11 & 5,19E-14 & 0,04 & $1,32 \mathrm{E}-14$ & 0,02 \\
\hline${ }^{51} \mathrm{Cr}$ & $4,00 \mathrm{E}-07$ & $8,23 E-15$ & 0,01 & $6,27 \mathrm{E}-15$ & 0,00 & $4,69 \mathrm{E}-15$ & 0,01 \\
\hline${ }^{54} \mathrm{Mn}$ & $2,20 \mathrm{E}-06$ & $3,20 \mathrm{E}-12$ & 2,62 & $1,44 \mathrm{E}-12$ & 1,05 & $1,01 \mathrm{E}-12$ & 1,23 \\
\hline${ }^{59} \mathrm{Fe}$ & 1,60E-06 & $2,24 \mathrm{E}-12$ & 1,84 & $2,71 \mathrm{E}-12$ & 1,98 & $2,50 \mathrm{E}-12$ & 3,03 \\
\hline $58 \mathrm{Co}$ & $6,00 \mathrm{E}-07$ & $5,05 \mathrm{E}-13$ & 0,41 & $5,37 E-13$ & 0,39 & $4,50 \mathrm{E}-13$ & 0,55 \\
\hline${ }^{60} \mathrm{Co}$ & $2,80 \mathrm{E}-06$ & $3,02 \mathrm{E}-11$ & 24,76 & $3,53 \mathrm{E}-11$ & 25,79 & $2,72 \mathrm{E}-11$ & 33,01 \\
\hline${ }^{95} \mathrm{Zr}$ & 4,00E-07 & 7,96E-13 & 0,65 & $9,70 \mathrm{E}-13$ & 0,71 & $9,00 \mathrm{E}-13$ & 1,09 \\
\hline${ }^{95} \mathrm{Nb}$ & $2,00 \mathrm{E}-06$ & $1,25 \mathrm{E}-12$ & 1,02 & $1,46 \mathrm{E}-12$ & 1,07 & $1,32 \mathrm{E}-12$ & 1,60 \\
\hline${ }^{131} \mid$ & $3,00 \mathrm{E}-07$ & $1,66 \mathrm{E}-12$ & 1,36 & $2,59 \mathrm{E}-13$ & 0,19 & $1,48 \mathrm{E}-14$ & 0,02 \\
\hline${ }^{134} \mathrm{Cs}$ & $4,00 \mathrm{E}-07$ & $2,89 \mathrm{E}-12$ & 2,37 & $3,49 \mathrm{E}-12$ & 2,55 & $2,86 \mathrm{E}-12$ & 3,47 \\
\hline${ }^{13 /} \mathrm{Cs}$ & 1,40E-06 & $1,55 \mathrm{E}-11$ & 12,71 & $2,26 \mathrm{E}-11$ & 16,51 & $1,77 \mathrm{E}-11$ & 21,48 \\
\hline${ }^{94} \mathrm{Nb}$ & $2,00 \mathrm{E}-07$ & $2,82 \mathrm{E}-12$ & 2,31 & $3,92 \mathrm{E}-12$ & 2,86 & $2,72 \mathrm{E}-12$ & 3,30 \\
\hline${ }^{65} \mathrm{Zn}$ & $6,00 \mathrm{E}-07$ & $1,16 \mathrm{E}-12$ & 0,95 & $6,58 \mathrm{E}-13$ & 0,48 & $2,89 \mathrm{E}-13$ & 0,35 \\
\hline${ }^{90} \mathrm{Sr}$ & 1,11E-07 & $4,51 \mathrm{E}-12$ & 3,70 & $7,26 \mathrm{E}-12$ & 5,30 & 5,78E-12 & 7,01 \\
\hline${ }^{239} \mathrm{Pu}$ & 1,90E-09 & $3,89 \mathrm{E}-11$ & 31,89 & $4,01 \mathrm{E}-11$ & 29,30 & $1,39 \mathrm{E}-11$ & 16,87 \\
\hline $\begin{array}{l}{ }^{238} \mathrm{Pu} \\
\mathrm{LAR}\end{array}$ & $8,00 \mathrm{E}-10$ & $\begin{array}{l}1,62 \mathrm{E}-11 \\
1,22 \mathrm{E}-10\end{array}$ & 13,28 & $\begin{array}{l}1,61 \mathrm{E}-11 \\
1,37 \mathrm{E}-10\end{array}$ & 11,76 & $\begin{array}{l}5,79 \mathrm{E}-12 \\
8,25 \mathrm{E}-11\end{array}$ & 7,03 \\
\hline \multicolumn{8}{|c|}{ Пероральное поступление с питьевой водой } \\
\hline $\begin{array}{l}{ }^{1377} \mathrm{Cs} \\
{ }^{60} \mathrm{Co} \\
\text { LAR }\end{array}$ & $\begin{array}{l}1,00 \mathrm{E}-02 \\
1,00 \mathrm{E}-02\end{array}$ & $\begin{array}{l}3,93 \mathrm{E}-09 \\
6,28 \mathrm{E}-09 \\
1,02 \mathrm{E}-08 \\
\end{array}$ & \begin{tabular}{|l|}
38,49 \\
61,51
\end{tabular} & $\begin{array}{l}5,26 \mathrm{E}-09 \\
1,21 \mathrm{E}-09 \\
6,47 \mathrm{E}-09 \\
\end{array}$ & $\begin{array}{l}81,30 \\
18,70\end{array}$ & $\begin{array}{l}2,45 \mathrm{E}-09 \\
5,01 \mathrm{E}-10 \\
2,95 \mathrm{E}-09 \\
\end{array}$ & $\begin{array}{l}83,02 \\
16,98\end{array}$ \\
\hline \multicolumn{8}{|c|}{ Пероральное поступление с пищей } \\
\hline $\begin{array}{c}{ }^{137} \mathrm{Cs} \\
\text { LAR } \\
\Sigma \text { LAR }\end{array}$ & 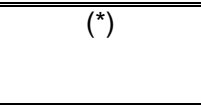 & $\begin{array}{l}3,32 \mathrm{E}-07 \\
3,32 \mathrm{E}-07 \\
\text { 3,42E-07 }\end{array}$ & 100 & $\begin{array}{l}5,22 \mathrm{E}-07 \\
5,22 \mathrm{E}-07 \\
5,29 \mathrm{E}-07 \\
\end{array}$ & 100 & $\begin{array}{l}2,43 \mathrm{E}-07 \\
2,43 \mathrm{E}-07 \\
\mathbf{2 , 4 6 E - 0 7}\end{array}$ & 100 \\
\hline
\end{tabular}

$\left(^{*}\right)$ Удельная активность ${ }^{137} \mathrm{Cs}$ в пищевых продуктах (Бк/кг): грибы - 1,4, картофель - 1,1, рыба - 1,9, овес - 1,3, мясо 0,8, молоко - 0,7 . 
Таблица 12

Пожизненные атрибутивные риски (LAR) онкозаболеваемости после ингаляционного и перорального поступления с питьевой водой и пищей в течение года в организм суммарного набора радионуклидов и отдельных радионуклидов от выбросов и сбросов Курской АЭС за 2016 г. (возраст при поступлении радионуклидов: 5 лет, 20 лет, 50 лет; женщины)

\begin{tabular}{|c|c|c|c|c|c|c|c|}
\hline $\begin{array}{l}\text { Радио- } \\
\text { нуклид }\end{array}$ & $\begin{array}{c}\text { Среднегодовая } \\
\text { объёмная } \\
\text { активность } \\
\text { радионуклида } \\
\text { в приземном } \\
\text { слое атмо- } \\
\text { сферного воз- } \\
\text { духа (Бк/м³) и } \\
\text { питьевой воде } \\
\text { (Бк/л) }\end{array}$ & $\begin{array}{c}\text { LAR } \\
\text { (5 лет) }\end{array}$ & $\begin{array}{c}\text { Вклад радио- } \\
\text { нуклида в LAR } \\
\text { (5 лет), \% }\end{array}$ & $\begin{array}{c}\text { LAR } \\
\text { (20 лет) }\end{array}$ & $\begin{array}{c}\text { Вклад радио- } \\
\text { нуклида в LAR } \\
\text { (20 лет), \% }\end{array}$ & $\begin{array}{c}\text { LAR } \\
(50 \text { лет) }\end{array}$ & $\begin{array}{c}\text { Вклад радио- } \\
\text { нуклида в LAR } \\
\text { (50 лет), \% }\end{array}$ \\
\hline \multicolumn{8}{|c|}{ Ингаляционное поступление } \\
\hline${ }^{24} \mathrm{Na}$ & 1,00E-07 & $1,85 \mathrm{E}-13$ & 0,09 & $7,23 E-14$ & 0,03 & $1,70 \mathrm{E}-14$ & 0,01 \\
\hline${ }^{51} \mathrm{Cr}$ & $4,00 \mathrm{E}-07$ & $1,59 \mathrm{E}-14$ & 0,01 & $1,16 \mathrm{E}-14$ & 0,01 & $8,00 \mathrm{E}-15$ & 0,01 \\
\hline${ }^{54} \mathrm{Mn}$ & $2,20 \mathrm{E}-06$ & $6,07 E-12$ & 2,84 & $2,90 \mathrm{E}-12$ & 1,36 & $1,75 \mathrm{E}-12$ & 1,28 \\
\hline${ }^{59} \mathrm{Fe}$ & 1,60E-06 & $4,73 E-12$ & 2,21 & $5,35 \mathrm{E}-12$ & 2,51 & $4,52 \mathrm{E}-12$ & 3,29 \\
\hline $58 \mathrm{Co}$ & $6,00 \mathrm{E}-07$ & $1,23 \mathrm{E}-12$ & 0,58 & $1,11 \mathrm{E}-12$ & 0,52 & $8,16 \mathrm{E}-13$ & 0,59 \\
\hline${ }^{60} \mathrm{Co}$ & $2,80 \mathrm{E}-06$ & $7,82 \mathrm{E}-11$ & 36,58 & $7,49 \mathrm{E}-11$ & 35,14 & $5,14 \mathrm{E}-11$ & 37,47 \\
\hline${ }^{95} \mathrm{Zr}$ & 4,00E-07 & $1,77 \mathrm{E}-12$ & 0,83 & $1,94 \mathrm{E}-12$ & 0,91 & $1,64 \mathrm{E}-12$ & 1,20 \\
\hline${ }^{95} \mathrm{Nb}$ & $2,00 \mathrm{E}-06$ & $2,71 \mathrm{E}-12$ & 1,27 & $2,89 \mathrm{E}-12$ & 1,36 & $2,37 \mathrm{E}-12$ & 1,73 \\
\hline${ }^{131} \mid$ & $3,00 \mathrm{E}-07$ & $1,83 \mathrm{E}-11$ & 8,56 & $2,69 \mathrm{E}-12$ & 1,26 & $8,75 \mathrm{E}-14$ & 0,06 \\
\hline${ }^{134} \mathrm{Cs}$ & $4,00 \mathrm{E}-07$ & $6,99 \mathrm{E}-12$ & 3,27 & $7,19 \mathrm{E}-12$ & 3,37 & $5,38 \mathrm{E}-12$ & 3,92 \\
\hline${ }^{13 /} \mathrm{Cs}$ & 1,40E-06 & $3,38 \mathrm{E}-11$ & 15,81 & $4,50 \mathrm{E}-11$ & 21,11 & $3,46 \mathrm{E}-11$ & 25,22 \\
\hline${ }^{94} \mathrm{Nb}$ & $2,00 \mathrm{E}-07$ & $6,76 \mathrm{E}-12$ & 3,16 & $8,04 \mathrm{E}-12$ & 3,77 & $5,36 \mathrm{E}-12$ & 3,91 \\
\hline${ }^{65} \mathrm{Zn}$ & $6,00 \mathrm{E}-07$ & $2,01 \mathrm{E}-12$ & 0,94 & $1,05 \mathrm{E}-12$ & 0,49 & $3,96 \mathrm{E}-13$ & 0,29 \\
\hline${ }^{90} \mathrm{Sr}$ & 1,11E-07 & $9,09 \mathrm{E}-12$ & 4,25 & $1,41 \mathrm{E}-11$ & 6,62 & $1,14 \mathrm{E}-11$ & 8,31 \\
\hline${ }^{239} \mathrm{Pu}$ & 1,90E-09 & $2,97 \mathrm{E}-11$ & 13,89 & $3,29 E-11$ & 15,44 & $1,23 \mathrm{E}-11$ & 8,97 \\
\hline $\begin{array}{l}{ }^{238} \mathrm{Pu} \\
\mathrm{LAR}\end{array}$ & $8,00 \mathrm{E}-10$ & $\begin{array}{l}1,22 \mathrm{E}-11 \\
2,14 \mathrm{E}-10\end{array}$ & 5,71 & $\begin{array}{l}1,30 \mathrm{E}-11 \\
2,13 \mathrm{E}-10\end{array}$ & 6,10 & $\begin{array}{l}5,14 \mathrm{E}-12 \\
1,37 \mathrm{E}-10\end{array}$ & 3,75 \\
\hline \multicolumn{8}{|c|}{ Пероральное поступление с питьевой водой } \\
\hline $\begin{array}{l}{ }^{1377} \mathrm{Cs} \\
{ }^{60} \mathrm{Co} \\
\text { LAR }\end{array}$ & $\begin{array}{l}1,00 \mathrm{E}-02 \\
1,00 \mathrm{E}-02\end{array}$ & $\begin{array}{l}7,54 \mathrm{E}-09 \\
1,06 \mathrm{E}-08 \\
1,81 \mathrm{E}-08 \\
\end{array}$ & \begin{tabular}{|l|}
41,57 \\
58,43
\end{tabular} & $\begin{array}{l}9,22 \mathrm{E}-09 \\
1,58 \mathrm{E}-09 \\
1,08 \mathrm{E}-08 \\
\end{array}$ & $\begin{array}{l}85,37 \\
14,63\end{array}$ & $\begin{array}{l}3,55 \mathrm{E}-09 \\
5,97 \mathrm{E}-10 \\
4,15 \mathrm{E}-09 \\
\end{array}$ & $\begin{array}{l}85,60 \\
14,40\end{array}$ \\
\hline \multicolumn{8}{|c|}{ Пероральное поступление с пищей } \\
\hline $\begin{array}{c}{ }^{137} \mathrm{Cs} \\
\text { LAR } \\
\Sigma \text { LAR }\end{array}$ & 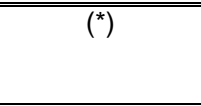 & $\begin{array}{l}6,37 \mathrm{E}-07 \\
6,37 \mathrm{E}-07 \\
6,56 \mathrm{E}-07\end{array}$ & 100 & $\begin{array}{l}9,16 \mathrm{E}-07 \\
9,16 \mathrm{E}-07 \\
\mathbf{9 , 2 7 E}-07\end{array}$ & 100 & $\begin{array}{l}3,52 \mathrm{E}-07 \\
3,52 \mathrm{E}-07 \\
3,57 \mathrm{E}-07\end{array}$ & 100 \\
\hline
\end{tabular}

$\left(^{*}\right)$ Удельная активность ${ }^{137} \mathrm{Cs}$ в пищевых продуктах (Бк/кг): грибы - 1,4, картофель - 1,1, рыба - 1,9, овес - 1,3, мясо 0,8, молоко $-0,7$. 
Таблица 13

Пожизненные атрибутивные риски (LAR) онкозаболеваемости после ингаляционного и перорального поступления с питьевой водой и пищей в течение года в организм суммарного набора радионуклидов и отдельных радионуклидов от выбросов и сбросов Ленинградской АЭС за 2016 г. (возраст при поступлении радионуклидов: 5 лет, 20 лет, 50 лет; мужчины)

\begin{tabular}{|c|c|c|c|c|c|c|c|}
\hline $\begin{array}{l}\text { Радио- } \\
\text { нуклид }\end{array}$ & \begin{tabular}{|c|} 
Среднегодовая \\
объёмная актив- \\
ность радионук- \\
лида в призем- \\
ном слое атмо- \\
сферного возду- \\
ха $\left(\right.$ Бк/м $/$ 3 $\left.^{3}\right)$ в \\
питевой воде \\
$(Б / / л)$ \\
\end{tabular} & $\begin{array}{l}\text { LAR } \\
\text { (5 лет) }\end{array}$ & $\begin{array}{c}\text { Вклад радио- } \\
\text { нуклида в LAR } \\
\text { (5 лет), \% }\end{array}$ & $\begin{array}{c}\text { LAR } \\
\text { (20 лет) }\end{array}$ & $\begin{array}{c}\text { Вклад радио- } \\
\text { нуклида в LAR } \\
\text { (20 лет), \% }\end{array}$ & $\begin{array}{c}\text { LAR } \\
\text { (50 лет) }\end{array}$ & $\begin{array}{c}\text { Вклад радио- } \\
\text { нуклида в LAR } \\
\text { (50 лет), \% }\end{array}$ \\
\hline 1 & 2 & 3 & 4 & 5 & 6 & 7 & 8 \\
\hline \multicolumn{8}{|c|}{ "Ингаляционное поступление } \\
\hline$\overline{{ }^{137} \mathrm{Cs}}$ & 3,30E-05 & 3,34E-10 & 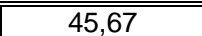 & $4,4,75 \mathrm{E}-10$ & 63,75 & $3,80 \mathrm{E}-10$ & 64,81 \\
\hline${ }^{90} \mathrm{Sr}$ & $8,50 \mathrm{E}-08$ & $3,14 \mathrm{E}-12$ & 0,43 & $4,94 \mathrm{E}-12$ & 0,66 & $4,04 \mathrm{E}-12$ & 0,69 \\
\hline${ }^{7} \mathrm{Be}$ & $2,96 \mathrm{E}-03$ & $1,67 \mathrm{E}-10$ & 22,84 & $6,15 \mathrm{E}-11$ & 8,25 & $4,57 \mathrm{E}-11$ & 7,79 \\
\hline${ }^{134} \mathrm{Cs}$ & $8,50 \mathrm{E}-06$ & $5,62 \mathrm{E}-11$ & 7,68 & $6,64 \mathrm{E}-11$ & 8,91 & $5,50 \mathrm{E}-11$ & 9,38 \\
\hline${ }^{60} \mathrm{Co}$ & $1,10 \mathrm{E}-05$ & $1,09 \mathrm{E}-10$ & 14,90 & $1,25 \mathrm{E}-10$ & 16,78 & $9,77 \mathrm{E}-11$ & 16,66 \\
\hline $54 \mathrm{Mn}$ & $7,20 \mathrm{E}-06$ & $8,96 \mathrm{E}-12$ & 1,23 & $4,31 \mathrm{E}-12$ & 0,58 & $3,04 \mathrm{E}-12$ & 0,52 \\
\hline${ }^{51} \mathrm{Cr}$ & $3,20 \mathrm{E}-05$ & $6,22 \mathrm{E}-13$ & 0,09 & $4,60 \mathrm{E}-13$ & 0,06 & $3,44 \mathrm{E}-13$ & 0,06 \\
\hline${ }^{131} \mid$ & $6,00 \mathrm{E}-06$ & $5,24 \mathrm{E}-11$ & 7,17 & $7,48 \mathrm{E}-12$ & 1,00 & $5,30 \mathrm{E}-13$ & 0,09 \\
\hline LAR & & 7,31E-10 & & $7,45 \mathrm{E}-10$ & & $5,86 \mathrm{E}-10$ & \\
\hline \multicolumn{8}{|c|}{ Пероральное поступление с питьевой водой } \\
\hline${ }^{13 /} \mathrm{Cs}$ & $\overline{1,20 \mathrm{E}-02}$ & $\overline{4,34 \mathrm{E}-09}$ & 4,62 & 5,77E-09 & 10,72 & $2,79 \mathrm{E}-09$ & 10,82 \\
\hline${ }^{60} \mathrm{Co}$ & $1,20 \mathrm{E}-02$ & $7,03 \mathrm{E}-09$ & 7,49 & $1,37 \mathrm{E}-09$ & 2,54 & $5,97 \mathrm{E}-10$ & 2,32 \\
\hline${ }^{3} \mathrm{H}$ & $3,00 \mathrm{E}+01$ & $8,25 \mathrm{E}-08$ & 87,89 & 4,67E-08 & 86,74 & $2,24 \mathrm{E}-08$ & 86,87 \\
\hline \multirow{2}{*}{\multicolumn{8}{|c|}{ "Пероральное поступление с пищей }} \\
\hline & & & & & & & \\
\hline $\begin{array}{l}{ }^{13 /} \mathrm{Cs} \\
\text { LAR } \\
\Sigma \text { LAR }\end{array}$ & 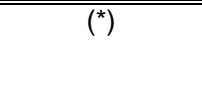 & $\begin{array}{l}2,04 \mathrm{E}-07 \\
2,04 \mathrm{E}-07 \\
\mathbf{2 , 9 9 E - 0 7}\end{array}$ & 100 & $\begin{array}{l}5,77 \mathrm{E}-07 \\
5,77 \mathrm{E}-07 \\
6,32 \mathrm{E}-07\end{array}$ & 100 & $\begin{array}{l}2,79 E-07 \\
2,79 E-07 \\
3,05 E-07\end{array}$ & 100 \\
\hline
\end{tabular}

$\left({ }^{*}\right)$ Удельная активность ${ }^{137} \mathrm{Cs}$ в пищевых продуктах (Бк/кг): картофель - 0,3, ягоды - 5,1, грибы - 66, рыба - 6,4, молоко - 0,08.

Таблица 14

Пожизненные атрибутивные риски (LAR) онкозаболеваемости после ингаляционного и перорального поступления с питьевой водой и пищей в течение года в организм суммарного набора радионуклидов и отдельных радионуклидов от выбросов и сбросов Ленинградской АЭС за 2016 г. (возраст при поступлении радионуклидов: 5 лет, 20 лет, 50 лет; женщины)

\begin{tabular}{|c|c|c|c|c|c|c|c|}
\hline 1 & 2 & 3 & 4 & 5 & 6 & 7 & 8 \\
\hline \multicolumn{8}{|c|}{ Ингаляционное поступление } \\
\hline${ }^{13 /} \mathrm{Cs}$ & 3,30E-05 & $7,45 \mathrm{E}-10$ & 34,54 & 9,72E-10 & 59,44 & $7,28 \mathrm{E}-10$ & 65,54 \\
\hline${ }^{90} \mathrm{Sr}$ & $8,50 \mathrm{E}-08$ & $6,33 \mathrm{E}-12$ & 0,29 & $9,77 \mathrm{E}-12$ & 0,60 & $7,80 \mathrm{E}-12$ & 0,70 \\
\hline${ }^{7} \mathrm{Be}$ & 2,96E-03 & $3,50 \mathrm{E}-10$ & 16,23 & $1,38 \mathrm{E}-10$ & 8,44 & $8,12 \mathrm{E}-11$ & 7,31 \\
\hline${ }^{134} \mathrm{Cs}$ & $8,50 \mathrm{E}-06$ & $1,44 \mathrm{E}-10$ & 6,68 & $1,42 \mathrm{E}-10$ & 8,68 & $1,03 E-10$ & 9,27 \\
\hline${ }^{60} \mathrm{Co}$ & $1,10 \mathrm{E}-05$ & $3,03 E-10$ & 14,05 & $2,77 \mathrm{E}-10$ & 16,94 & $1,82 \mathrm{E}-10$ & 16,38 \\
\hline $54 \mathrm{Mn}$ & $7,20 \mathrm{E}-06$ & $1,94 \mathrm{E}-11$ & 0,90 & $9,14 \mathrm{E}-12$ & 0,56 & $5,23 \mathrm{E}-12$ & 0,47 \\
\hline${ }^{51} \mathrm{Cr}$ & $3,20 \mathrm{E}-05$ & $1,27 \mathrm{E}-12$ & 0,06 & $8,81 \mathrm{E}-13$ & 0,05 & $5,81 \mathrm{E}-13$ & 0,05 \\
\hline${ }^{131}$ & $6,00 \mathrm{E}-06$ & $5,88 \mathrm{E}-10$ & 27,26 & $8,66 \mathrm{E}-11$ & 5,30 & $3,04 \mathrm{E}-12$ & 0,27 \\
\hline LAR & & $2,16 \mathrm{E}-09$ & & 1,64E-09 & & 1,11E-09 & \\
\hline \multicolumn{8}{|c|}{ " Пероральное поступление с питьевой водой } \\
\hline 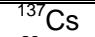 & $1,20 \mathrm{E}-02$ & $9,66 \mathrm{E}-09$ & 4,31 & $1,12 \mathrm{E}-08$ & 10,35 & $4,10 \mathrm{E}-09$ & 10,59 \\
\hline 㟶 $\mathrm{Co}$ & $1,20 \mathrm{E}-02$ & $1,35 \mathrm{E}-08$ & 6,02 & 1,93E-09 & 1,78 & $7,05 \mathrm{E}-10$ & 1,82 \\
\hline${ }^{3} \mathrm{H}$ & $3,00 \mathrm{E}+01$ & $2,01 \mathrm{E}-07$ & 89,67 & $9,51 \mathrm{E}-08$ & 87,87 & $3,39 \mathrm{E}-08$ & 87,59 \\
\hline LAR & & $2,24 \mathrm{E}-07$ & & $1,08 \mathrm{E}-07$ & & $3,87 \mathrm{E}-08$ & \\
\hline \multicolumn{8}{|c|}{ Пероральное поступление с пищей } \\
\hline${ }^{13 /} \mathrm{Cs}$ & $\left({ }^{*}\right)$ & $4,55 \mathrm{E}-07$ & 100 & $1,12 \mathrm{E}-06$ & 100 & $4,10 \mathrm{E}-07$ & 100 \\
\hline $\begin{array}{c}\text { LAR } \\
\Sigma \text { LAR }\end{array}$ & & $\begin{array}{l}\text { 4,55E-07 } \\
\mathbf{6 , 8 1 E - 0 7}\end{array}$ & & $\begin{array}{l}1,12 \mathrm{E}-06 \\
\mathbf{1 , 2 3 E}-06\end{array}$ & & $\begin{array}{l}4,10 \mathrm{E}-07 \\
\mathbf{4 , 5 0 E - 0 7}\end{array}$ & \\
\hline
\end{tabular}

(*) Удельная активность ${ }^{137}$ Cs в пищевых продуктах (Бк/кг): картофель - 0,3, ягоды - 5,1, грибы - 66, рыба - 6,4, молоко - 0,08. 
Таблица 15

Пожизненные атрибутивные риски (LAR) онкозаболеваемости после ингаляционного и перорального поступления с питьевой водой и пищей в течение года в организм суммарного набора радионуклидов и отдельных радионуклидов от выбросов и сбросов Нововоронежской АЭС за 2016 г. (возраст при поступлении радионуклидов: 5 лет, 20 лет, 50 лет; мужчины)

\begin{tabular}{|c|c|c|c|c|c|c|c|}
\hline $\begin{array}{l}\text { Радио- } \\
\text { нуклид }\end{array}$ & \begin{tabular}{|c|} 
Среднегодовая \\
объёмная \\
активность \\
радионуклида \\
в приземном \\
слое атмо- \\
сферного воз- \\
духа (Бк/м $\left.{ }^{3}\right)$ и \\
питьевой воде \\
$($ Бк/л) \\
\end{tabular} & $\begin{array}{l}\text { LAR } \\
\text { (5 лет) }\end{array}$ & \begin{tabular}{|c} 
Вклад радио- \\
нуклида в LAR \\
(5 лет), \%
\end{tabular} & $\begin{array}{c}\text { LAR } \\
\text { (20 лет) }\end{array}$ & $\begin{array}{c}\text { Вклад радио- } \\
\text { нуклида в LAR } \\
\text { (20 лет), \% }\end{array}$ & $\begin{array}{c}\text { LAR } \\
\text { (50 лет) }\end{array}$ & $\begin{array}{c}\text { Вклад радио- } \\
\text { нуклида в LAR } \\
\text { (50 лет), \% }\end{array}$ \\
\hline$\overline{11}$ & 2 & $\overline{3}$ & 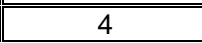 & 5 & 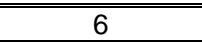 & $\overline{7}$ & 8 \\
\hline \multicolumn{8}{|c|}{ Ингаляционное поступление } \\
\hline${ }^{54} \mathrm{Mn}$ & $2,50 \mathrm{E}-07$ & 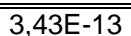 & 0,67 & $1,56 \mathrm{E}-13$ & 0,23 & 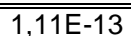 & 0,21 \\
\hline${ }^{58} \mathrm{Co}$ & $1,05 \mathrm{E}-06$ & $8,46 \mathrm{E}-13$ & 1,64 & $8,91 \mathrm{E}-13$ & 1,31 & $7,55 \mathrm{E}-13$ & 1,40 \\
\hline${ }^{60} \mathrm{Co}$ & $1,85 \mathrm{E}-06$ & $1,91 \mathrm{E}-11$ & 37,10 & $2,21 \mathrm{E}-11$ & 32,43 & $1,73 \mathrm{E}-11$ & 32,12 \\
\hline${ }^{13 /} \mathrm{Cs}$ & $2,95 \mathrm{E}-06$ & $3,12 \mathrm{E}-11$ & 60,60 & $4,50 \mathrm{E}-11$ & 66,03 & $3,57 \mathrm{E}-11$ & 66,28 \\
\hline LAR & & $5,15 \mathrm{E}-11$ & & $6,81 \mathrm{E}-11$ & & $5,39 \mathrm{E}-11$ & \\
\hline \multicolumn{8}{|c|}{ Пероральное поступление с питьевой водой } \\
\hline${ }^{137} \mathrm{Cs}$ & 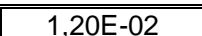 & 4,55E-09 & 34,97 & 6.05E-09 & 78,98 & $2,87 \mathrm{E}-09$ & 80,89 \\
\hline${ }^{60} \mathrm{Co}$ & $1,40 \mathrm{E}-02$ & $8,46 \mathrm{E}-09$ & 65,03 & $1,61 \mathrm{E}-09$ & 21,02 & $6,78 \mathrm{E}-10$ & 19,11 \\
\hline LAR & & $1,30 \mathrm{E}-08$ & & 7,66E-09 & & 3,55E-09 & \\
\hline \multicolumn{8}{|c|}{ Пероральное поступление с пищей } \\
\hline${ }^{137} \mathrm{Cs}$ & 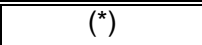 & $2,10 \mathrm{E}-08$ & 100 & $2,35 \mathrm{E}-08$ & 100 & $1,11 \mathrm{E}-08$ & 100 \\
\hline $\begin{array}{c}\text { LAR } \\
\Sigma \text { LAR }\end{array}$ & & $\begin{array}{l}2,10 \mathrm{E}-08 \\
\mathbf{3 , 4 1 E}-08\end{array}$ & & $\begin{array}{l}2,35 \mathrm{E}-08 \\
\mathbf{3 , 1 2 E}-08\end{array}$ & & $\begin{array}{l}1,11 \mathrm{E}-08 \\
\mathbf{1 , 4 7 E - 0 8}\end{array}$ & \\
\hline
\end{tabular}

$\left(^{*}\right)$ Удельная активность ${ }^{137} \mathrm{Cs}$ в пищевых продуктах (Бк/кг): мясо - 0,1, рыба - 0,09, пшеница - 0,07, молоко - 0,07.

Таблица 16

Пожизненные атрибутивные риски (LAR) онкозаболеваемости после ингаляционного и перорального поступления с питьевой водой и пищей в течение года в организм суммарного набора радионуклидов и отдельных радионуклидов от выбросов и сбросов Нововоронежской АЭС за 2016 г. (возраст при поступлении радионуклидов: 5 лет, 20 лет, 50 лет; женщины)

\begin{tabular}{|c|c|c|c|c|c|c|c|}
\hline 1 & 2 & 3 & 4 & 5 & 6 & 7 & 8 \\
\hline \multicolumn{8}{|c|}{ Ингаляционное поступление } \\
\hline${ }^{54} \mathrm{Mn}$ & $2,50 \mathrm{E}-07$ & $6,76 \mathrm{E}-13$ & 0,56 & (3,15E-13 & 0,24 & $1,81 \mathrm{E}-13$ & 0,19 \\
\hline${ }^{58} \mathrm{Co}$ & 1,05E-06 & $2,11 \mathrm{E}-12$ & 1,76 & 1,81E-12 & 1,35 & $1,28 \mathrm{E}-12$ & 1,32 \\
\hline${ }^{60} \mathrm{Co}$ & $1,85 \mathrm{E}-06$ & $5,06 \mathrm{E}-11$ & 42,28 & $4,61 \mathrm{E}-11$ & 34,42 & $3,06 \mathrm{E}-11$ & 31,46 \\
\hline${ }^{13 /} \mathrm{Cs}$ & 2,95E-06 & $6,63 E-11$ & 55,39 & $8,57 \mathrm{E}-11$ & 63,99 & $6,52 \mathrm{E}-11$ & 67,04 \\
\hline LAR & & $1,20 \mathrm{E}-10$ & & $1,34 \mathrm{E}-10$ & & $9,73 \mathrm{E}-11$ & \\
\hline \multicolumn{8}{|c|}{ "Пероральное поступление с питьевой водой } \\
\hline${ }^{137} \mathrm{Cs}$ & 1,20E-02 & 9,52E-09 & 38,05 & $1,11 \mathrm{E}-08$ & 83,40 & 4,07E-09 & 83,49 \\
\hline${ }^{60} \mathrm{Co}$ & 1,40E-02 & $1,55 \mathrm{E}-08$ & 61,95 & 2,21E-09 & 16,60 & $8,05 \mathrm{E}-10$ & 16,51 \\
\hline LAR & & $2,50 \mathrm{E}-08$ & & $1,33 \mathrm{E}-08$ & & $4,88 \mathrm{E}-09$ & \\
\hline \multicolumn{8}{|c|}{ Пероральное поступление с пищей } \\
\hline${ }^{137} \mathrm{Cs}$ & $\left(\left(^{*}\right)\right.$ & 4,39E-08 & 100 & $4,31 \mathrm{E}-08$ & 100 & 1,58E-08 & 100 \\
\hline LAR & & $4,39 \mathrm{E}-08$ & & 4,31E-08 & & 1,58E-08 & \\
\hline$\Sigma$ LAR & & 6,91E-08 & & 5,66E-08 & & 2,08E-08 & \\
\hline
\end{tabular}

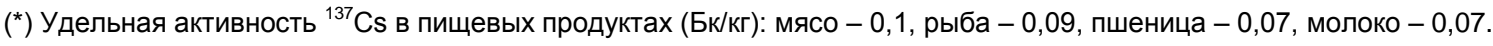


Таблица 17

Пожизненные атрибутивные риски (LAR) онкозаболеваемости после ингаляционного и перорального поступления с питьевой водой и пищей в течение года в организм суммарного набора радионуклидов и отдельных радионуклидов от выбросов и сбросов Ростовской АЭС за 2016 г. (возраст при поступлении радионуклидов: 5 лет, 20 лет, 50 лет; мужчины)

\begin{tabular}{|c|c|c|c|c|c|c|c|}
\hline $\begin{array}{l}\text { Радио- } \\
\text { нуклид }\end{array}$ & $\begin{array}{c}\text { Среднегодовая } \\
\text { объёмная } \\
\text { активность } \\
\text { радионуклида } \\
\text { в приземном } \\
\text { слое атмо- } \\
\text { сферного воз- } \\
\text { духа (Бк/м³) и } \\
\text { питьевой воде } \\
\text { (Бк/л) }\end{array}$ & $\begin{array}{c}\text { LAR } \\
\text { (5 лет) }\end{array}$ & $\begin{array}{c}\text { Вклад радио- } \\
\text { нуклида в LAR } \\
\text { (5 лет), \% }\end{array}$ & $\begin{array}{c}\text { LAR } \\
\text { (20 лет) }\end{array}$ & $\begin{array}{c}\text { Вклад радио- } \\
\text { нуклида в LAR } \\
\text { (20 лет), \% }\end{array}$ & $\begin{array}{c}\text { LAR } \\
\text { (50 лет) }\end{array}$ & $\begin{array}{c}\text { Вклад радио- } \\
\text { нуклида в LAR } \\
\text { (50 лет), \% }\end{array}$ \\
\hline \multicolumn{8}{|c|}{ Ингаляционное поступление } \\
\hline $\begin{array}{l}{ }^{173} \mathrm{Cs} \\
{ }^{90} \mathrm{Sr} \\
\text { LAR }\end{array}$ & $\begin{array}{l}2,40 \mathrm{E}-07 \\
4,90 \mathrm{E}-08\end{array}$ & $\begin{array}{l}2,57 \mathrm{E}-12 \\
1,93 \mathrm{E}-12 \\
4,50 \mathrm{E}-12\end{array}$ & $\begin{array}{l}57,11 \\
42,89\end{array}$ & $\begin{array}{l}3,71 \mathrm{E}-12 \\
3,07 \mathrm{E}-12 \\
6,78 \mathrm{E}-12\end{array}$ & $\begin{array}{l}54,72 \\
45,28\end{array}$ & $\begin{array}{l}3,00 \mathrm{E}-12 \\
2,53 \mathrm{E}-12 \\
5,53 \mathrm{E}-12\end{array}$ & $\begin{array}{l}54,25 \\
45,75\end{array}$ \\
\hline \multicolumn{8}{|c|}{ Пероральное поступление с питьевой водой } \\
\hline $\begin{array}{l}{ }^{137} \mathrm{Cs} \\
{ }^{60} \mathrm{Co} \\
{ }^{30} \mathrm{H} \\
\text { LAR }\end{array}$ & $\begin{array}{l}1,20 \mathrm{E}-02 \\
1,40 \mathrm{E}-02 \\
1,10 \mathrm{E}+00\end{array}$ & $\begin{array}{l}4,58 \mathrm{E}-09 \\
8,60 \mathrm{E}-09 \\
3,19 \mathrm{E}-09 \\
1,64 \mathrm{E}-08\end{array}$ & $\begin{array}{l}27,98 \\
52,54 \\
19,49\end{array}$ & $\begin{array}{l}6,12 \mathrm{E}-09 \\
1,70 \mathrm{E}-09 \\
1,82 \mathrm{E}-09 \\
9,64 \mathrm{E}-09\end{array}$ & $\begin{array}{r}63,49 \\
17,63 \\
18,88\end{array}$ & $\begin{array}{l}2,95 \mathrm{E}-09 \\
7,29 \mathrm{E}-10 \\
8,65 \mathrm{E}-10 \\
4,54 \mathrm{E}-09\end{array}$ & $\begin{array}{l}64,92 \\
16,04 \\
19,04\end{array}$ \\
\hline \multicolumn{8}{|c|}{ Пероральное поступление с пищей } \\
\hline $\begin{array}{c}{ }^{131} \mathrm{I} \\
\text { LAR } \\
\Sigma \text { LAR }\end{array}$ & $\left(\left(^{*}\right)\right.$ & $\begin{array}{l}8,64 \mathrm{E}-09 \\
8,64 \mathrm{E}-09 \\
2,50 \mathrm{E}-08\end{array}$ & 100 & $\begin{array}{l}5,39 \mathrm{E}-10 \\
5,39 \mathrm{E}-10 \\
\mathbf{1 , 0 2 E - 0 8}\end{array}$ & 100 & $\begin{array}{l}3,44 \mathrm{E}-11 \\
3,44 \mathrm{E}-11 \\
4,58 \mathrm{E}-09\end{array}$ & 100 \\
\hline
\end{tabular}

$\left(^{*}\right)$ Удельная активность ${ }^{131}$ І в пищевых продуктах (Бк/кг): молоко - 0,008.

Таблица 18

Пожизненные атрибутивные риски (LAR) онкозаболеваемости после ингаляционного и перорального поступления с питьевой водой и пищей в течение года в организм суммарного набора радионуклидов и отдельных радионуклидов от выбросов и сбросов Ростовской АЭС за 2016 г. (возраст при поступлении радионуклидов: 5 лет, 20 лет, 50 лет; женщины)

\begin{tabular}{|c|c|c|c|c|c|c|c|}
\hline $\begin{array}{l}\text { Радио- } \\
\text { нуклид }\end{array}$ & \begin{tabular}{|c|} 
Среднегодовая \\
объёмная \\
активность \\
радионуклида \\
в приземном \\
слое атмо- \\
сферного воз- \\
духа (Бк/м $\left.{ }^{3}\right)$ и \\
питьевой воде \\
(Бк/л) \\
\end{tabular} & $\begin{array}{c}\text { LAR } \\
\text { (5 лет) }\end{array}$ & $\begin{array}{c}\text { Вклад радио- } \\
\text { нуклида в LAR } \\
\text { (5 лет), \% }\end{array}$ & $\begin{array}{c}\text { LAR } \\
\text { (20 лет) }\end{array}$ & $\begin{array}{c}\text { Вклад радио- } \\
\text { нуклида в LAR } \\
\text { (20 лет), \% }\end{array}$ & $\begin{array}{c}\text { LAR } \\
\text { (50 лет) }\end{array}$ & $\begin{array}{c}\text { Вклад радио- } \\
\text { нуклида в LAR } \\
\text { (50 лет), \% }\end{array}$ \\
\hline \multicolumn{8}{|c|}{ Ингаляционное поступление } \\
\hline $\begin{array}{l}{ }^{137} \mathrm{Cs} \\
{ }^{90} \mathrm{Sr} \\
\mathrm{LAR} \\
\end{array}$ & $\begin{array}{l}2,40 \mathrm{E}-07 \\
4,90 \mathrm{E}-08\end{array}$ & $\begin{array}{l}5,53 \mathrm{E}-12 \\
3,77 \mathrm{E}-12 \\
9,30 \mathrm{E}-12 \\
\end{array}$ & $\begin{array}{l}59,46 \\
40,54\end{array}$ & $\begin{array}{l}7,26 \mathrm{E}-12 \\
5,81 \mathrm{E}-12 \\
1,31 \mathrm{E}-11 \\
\end{array}$ & $\begin{array}{l}55,55 \\
44,45\end{array}$ & $\begin{array}{l}5,50 \mathrm{E}-12 \\
4,68 \mathrm{E}-12 \\
1,02 \mathrm{E}-11 \\
\end{array}$ & $\begin{array}{l}54,03 \\
45,97\end{array}$ \\
\hline \multicolumn{8}{|c|}{ Пероральное поступление с питьевой водой } \\
\hline $\begin{array}{l}{ }^{13 /} \mathrm{Cs} \\
{ }^{60} \mathrm{Co} \\
{ }^{3} \mathrm{H} \\
\text { LAR }\end{array}$ & $\begin{array}{l}1,20 \mathrm{E}-02 \\
1,40 \mathrm{E}-02 \\
1,10 \mathrm{E}+00\end{array}$ & $\begin{array}{l}9,28 \mathrm{E}-09 \\
1,52 \mathrm{E}-08 \\
7,10 \mathrm{E}-09 \\
3,16 \mathrm{E}-08 \\
\end{array}$ & $\begin{array}{l}29,39 \\
48,13 \\
22,48\end{array}$ & $\begin{array}{l}1,12 \mathrm{E}-08 \\
2,27 \mathrm{E}-09 \\
3,48 \mathrm{E}-09 \\
1,70 \mathrm{E}-08 \\
\end{array}$ & $\begin{array}{l}66,08 \\
13,39 \\
20,53\end{array}$ & $\begin{array}{l}4,23 \mathrm{E}-09 \\
8,51 \mathrm{E}-10 \\
1,28 \mathrm{E}-09 \\
6,36 \mathrm{E}-09 \\
\end{array}$ & $\begin{array}{l}66,50 \\
13,38 \\
20,12\end{array}$ \\
\hline \multicolumn{8}{|c|}{ Пероральное поступление с пищей } \\
\hline $\begin{array}{c}{ }^{131} \mathrm{I} \\
\text { LAR } \\
\Sigma \text { LAR }\end{array}$ & $\left({ }^{*}\right)$ & $\begin{array}{l}1,10 \mathrm{E}-07 \\
1,10 \mathrm{E}-07 \\
\mathbf{1 , 4 2 E - 0 7}\end{array}$ & 100 & $\begin{array}{l}6,80 \mathrm{E}-09 \\
6,80 \mathrm{E}-09 \\
\mathbf{2 , 3 8 E - 0 8}\end{array}$ & 100 & $\begin{array}{l}2,97 \mathrm{E}-10 \\
2,97 \mathrm{E}-10 \\
\mathbf{6 , 6 6 E - 0 9}\end{array}$ & 100 \\
\hline
\end{tabular}

$\left({ }^{*}\right)$ Удельная активность ${ }^{131}$ І в пищевых продуктах (Бк/кг): молоко - 0,008. 
Таблица 19

Пожизненные атрибутивные риски (LAR) онкозаболеваемости после ингаляционного и перорального поступления с питьевой водой и пищей в течение года в организм суммарного набора радионуклидов и отдельных радионуклидов от выбросов и сбросов Смоленской АЭС за 2016 г. (возраст при поступлении радионуклидов: 5 лет, 20 лет, 50 лет; мужчины)

\begin{tabular}{|c|c|c|c|c|c|c|c|}
\hline $\begin{array}{l}\text { Радио- } \\
\text { нуклид }\end{array}$ & \begin{tabular}{|} 
Среднегодовая \\
объёмная \\
активность \\
радионуклида \\
в приземном \\
слое атмо- \\
сферного воз- \\
духа (Бк/м³ $)$ и \\
питьевой воде \\
(Бк/л) \\
\end{tabular} & $\begin{array}{l}\text { LAR } \\
\text { (5 лет) }\end{array}$ & \begin{tabular}{|c} 
Вклад радио- \\
нуклида в LAR \\
(5 лет), \%
\end{tabular} & $\begin{array}{c}\text { LAR } \\
\text { (20 лет) }\end{array}$ & \begin{tabular}{|c} 
Вклад радио- \\
нуклида в LAR \\
(20 лет), \%
\end{tabular} & $\begin{array}{c}\text { LAR } \\
\text { (50 лет) }\end{array}$ & $\begin{array}{c}\text { Вклад радио- } \\
\text { нуклида в LAR } \\
\text { (50 лет), \% }\end{array}$ \\
\hline \multicolumn{8}{|c|}{ Ингаляционное поступление } \\
\hline $\begin{array}{l}{ }^{13 /} \mathrm{Cs} \\
{ }^{134} \mathrm{Cs} \\
\text { LAR }\end{array}$ & $\begin{array}{l}7,80 \mathrm{E}-07 \\
1,30 \mathrm{E}-07\end{array}$ & $\begin{array}{l}8,21 \mathrm{E}-12 \\
8,83 \mathrm{E}-13 \\
9,09 \mathrm{E}-12\end{array}$ & $\begin{array}{c}90,29 \\
9,71\end{array}$ & $\begin{array}{l}1,18 \mathrm{E}-11 \\
1,06 \mathrm{E}-12 \\
1,29 \mathrm{E}-11\end{array}$ & $\begin{array}{l}91,76 \\
8,24\end{array}$ & $\begin{array}{l}9,28 \mathrm{E}-12 \\
8,70 \mathrm{E}-13 \\
1,02 \mathrm{E}-11\end{array}$ & $\begin{array}{l}91,43 \\
8,57\end{array}$ \\
\hline \multicolumn{8}{|c|}{ Пероральное поступление с питьевой водой } \\
\hline $\begin{array}{l}{ }^{60} \mathrm{Co} \\
{ }^{13 /} \mathrm{Cs} \\
\text { LAR }\end{array}$ & $\begin{array}{l}3,23 \mathrm{E}-06 \\
6,00 \mathrm{E}-03\end{array}$ & $\begin{array}{l}3,26 \mathrm{E}-11 \\
2,15 \mathrm{E}-09 \\
2,18 \mathrm{E}-09\end{array}$ & $\begin{array}{c}1,49 \\
98,51\end{array}$ & $\begin{array}{l}3,81 \mathrm{E}-11 \\
2,88 \mathrm{E}-09 \\
2,92 \mathrm{E}-09\end{array}$ & $\begin{array}{c}1,31 \\
98,69\end{array}$ & $\begin{array}{l}2,95 \mathrm{E}-11 \\
1,39 \mathrm{E}-09 \\
1,42 \mathrm{E}-09\end{array}$ & $\begin{array}{c}2,08 \\
97,92\end{array}$ \\
\hline \multicolumn{8}{|c|}{ Пероральное поступление с пищей } \\
\hline $\begin{array}{l}{ }^{137} \mathrm{Cs} \\
\text { LAR } \\
\Sigma \text { LAR }\end{array}$ & 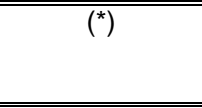 & $\begin{array}{l}6,93 \mathrm{E}-07 \\
6,93 \mathrm{E}-07 \\
\mathbf{6 , 9 5 E - 0 7} \\
\end{array}$ & 100 & $\begin{array}{l}8,04 \mathrm{E}-07 \\
8,04 \mathrm{E}-07 \\
8,07 \mathrm{E}-07 \\
\end{array}$ & 100 & $\begin{array}{l}3,89 \mathrm{E}-07 \\
3,89 \mathrm{E}-07 \\
3,90 \mathrm{E}-07 \\
\end{array}$ & 100 \\
\hline
\end{tabular}

(*) Удельная активность ${ }^{137} \mathrm{Cs}$ в пищевых продуктах (Бк/кг): грибы - 3, рыба - 1,8, овощи - 2,5, зерновые - 2,1, молоко - 2 .

Таблица 20

Пожизненные атрибутивные риски (LAR) онкозаболеваемости после ингаляционного и перорального поступления с питьевой водой и пищей в течение года в организм суммарного набора радионуклидов и отдельных радионуклидов от выбросов и сбросов Смоленской АЭС за 2016 г. (возраст при поступлении радионуклидов: 5 лет, 20 лет, 50 лет; женщины)

\begin{tabular}{|c|c|c|c|c|c|c|c|}
\hline $\begin{array}{l}\text { Радио- } \\
\text { нуклид }\end{array}$ & $\begin{array}{c}\text { Среднегодовая } \\
\text { объёмная } \\
\text { активность } \\
\text { радионуклида } \\
\text { в приземном } \\
\text { слое атмо- } \\
\text { сферного воз- } \\
\left.\text { духа (Бк/м }{ }^{3}\right) \text { и } \\
\text { питьевой воде } \\
(\text { Бк/л) } \\
\end{array}$ & $\begin{array}{l}\text { LAR } \\
\text { (5 лет) }\end{array}$ & $\begin{array}{c}\text { Вклад радио- } \\
\text { нуклида в LAR } \\
\text { (5 лет), \% }\end{array}$ & $\begin{array}{c}\text { LAR } \\
\text { (20 лет) }\end{array}$ & \begin{tabular}{|l} 
Вклад радио- \\
нуклида в LAR \\
(20 лет), \%
\end{tabular} & $\begin{array}{c}\text { LAR } \\
\text { (50 лет) }\end{array}$ & $\begin{array}{c}\text { Вклад радио- } \\
\text { нуклида в LAR } \\
\text { (50 лет), \% }\end{array}$ \\
\hline \multicolumn{8}{|c|}{ Ингаляционное поступление } \\
\hline $\begin{array}{l}{ }^{13 /} \mathrm{Cs} \\
{ }^{134} \mathrm{Cs} \\
\text { LAR }\end{array}$ & $\begin{array}{l}7,80 \mathrm{E}-07 \\
1,30 \mathrm{E}-07\end{array}$ & $\begin{array}{l}1,77 \mathrm{E}-11 \\
2,14 \mathrm{E}-12 \\
1,98 \mathrm{E}-11\end{array}$ & $\begin{array}{l}89,21 \\
10,79\end{array}$ & $\begin{array}{l}2,37 \mathrm{E}-11 \\
2,22 \mathrm{E}-12 \\
2,59 \mathrm{E}-11\end{array}$ & $\begin{array}{c}91,44 \\
8,56\end{array}$ & $\begin{array}{l}1,80 \mathrm{E}-11 \\
1,64 \mathrm{E}-12 \\
1,96 \mathrm{E}-11\end{array}$ & $\begin{array}{l}91,65 \\
8,35\end{array}$ \\
\hline \multicolumn{8}{|c|}{ " Пероральное поступление с питьевой водой } \\
\hline $\begin{array}{l}{ }^{60} \mathrm{Co} \\
{ }^{13 /} \mathrm{Cs} \\
\text { LAR } \\
\end{array}$ & $\begin{array}{l}3,23 \mathrm{E}-06 \\
6,00 \mathrm{E}-03\end{array}$ & $\begin{array}{l}8,48 \mathrm{E}-11 \\
4,15 \mathrm{E}-09 \\
4,23 \mathrm{E}-09 \\
\end{array}$ & $\begin{array}{c}2,00 \\
98,00\end{array}$ & $\begin{array}{l}8,21 \mathrm{E}-11 \\
5,24 \mathrm{E}-09 \\
5,32 \mathrm{E}-09 \\
\end{array}$ & $\begin{array}{c}1,54 \\
98,46\end{array}$ & $\begin{array}{l}5,58 \mathrm{E}-11 \\
2,08 \mathrm{E}-09 \\
2,14 \mathrm{E}-09 \\
\end{array}$ & $\begin{array}{c}2,61 \\
97,39\end{array}$ \\
\hline \multicolumn{8}{|c|}{ Пероральное поступление с пищей } \\
\hline $\begin{array}{l}{ }^{13 /} \mathrm{Cs} \\
\text { LAR } \\
\Sigma \text { LAR }\end{array}$ & $\left({ }^{*}\right)$ & $\begin{array}{l}\text { 1,34E-06 } \\
\text { 1,34E-06 } \\
\mathbf{1 , 3 4 E - 0 6}\end{array}$ & 100 & $\begin{array}{l}1,46 \mathrm{E}-06 \\
1,46 \mathrm{E}-06 \\
\mathbf{1 , 4 7 E - 0 6}\end{array}$ & 100 & $\begin{array}{l}5,79 \mathrm{E}-07 \\
5,79 \mathrm{E}-07 \\
\mathbf{5 , 8 1 E - 0 7}\end{array}$ & 100 \\
\hline
\end{tabular}

(*) Удельная активность ${ }^{137} \mathrm{Cs}$ в пищевых продуктах (Бк/кг): грибы - 3, рыба - 1,8, овощи - 2,5, зерновые - 2,1, молоко - 2. 
Таблица 21

Пожизненные атрибутивные риски (LAR) онкозаболеваемости от суммарного набора радионуклидов и отдельных радионуклидов от выбросов БРЕСТ-ОД-300 (возраст при поступлении радионуклидов: 5 лет, 20 лет, 50 лет; мужчины)

\begin{tabular}{|c|c|c|c|c|c|c|c|}
\hline $\begin{array}{l}\text { Радио- } \\
\text { нуклид }\end{array}$ & $\begin{array}{c}\text { Годовая } \\
\text { эффрективная } \\
\text { доза (мкЗв) }\end{array}$ & $\begin{array}{c}\text { LAR } \\
\text { (5 лет) }\end{array}$ & \begin{tabular}{|c|} 
Вклад радио- \\
нуклида в LAR \\
(5 лет), \% \\
\end{tabular} & $\begin{array}{c}\text { LAR } \\
\text { (20 лет) }\end{array}$ & \begin{tabular}{|c||} 
Вклад радио- \\
нуклида в LAR \\
(20 лет), \% \\
\end{tabular} & $\begin{array}{c}\text { LAR } \\
\text { (50 лет) }\end{array}$ & \begin{tabular}{|c} 
Вклад радио- \\
нуклида в LAR \\
(50 лет), \% \\
\end{tabular} \\
\hline \multicolumn{8}{|c|}{ Ингаляционное поступление } \\
\hline $\begin{array}{l}\text { Радиоак- } \\
\text { тивные } \\
\text { благород- }\end{array}$ & 0,11 & $1,11 \mathrm{E}-08$ & 3,52 & 7,77E-09 & 4,00 & 3,44E-09 & 3,89 \\
\hline $\begin{array}{c}\text { ные газы } \\
{ }^{14} \mathrm{C} \\
{ }^{3} \mathrm{H} \\
\mathrm{Pu}^{*} \\
{ }^{210} \mathrm{Po}\end{array}$ & $\begin{array}{l}1,22 \\
0,73 \\
0,49 \\
0,17\end{array}$ & $\begin{array}{l}1,17 \mathrm{E}-07 \\
7,11 \mathrm{E}-08 \\
2,16 \mathrm{E}-08 \\
4,49 \mathrm{E}-08\end{array}$ & $\begin{array}{c}37,06 \\
22,52 \\
6,84 \\
14,22\end{array}$ & $\begin{array}{l}7,78 \mathrm{E}-08 \\
4,68 \mathrm{E}-08 \\
1,13 \mathrm{E}-08 \\
2,79 \mathrm{E}-08\end{array}$ & $\begin{array}{c}40,07 \\
24,10 \\
5,82 \\
14,37\end{array}$ & $\begin{array}{l}3,88 \mathrm{E}-08 \\
2,29 \mathrm{E}-08 \\
3,55 \mathrm{E}-09 \\
9,48 \mathrm{E}-09\end{array}$ & $\begin{array}{c}43,86 \\
25,88 \\
4,01 \\
10,72\end{array}$ \\
\hline $\begin{array}{c}\text { Продукты } \\
\text { деления } \\
\mathbf{\Sigma} \text { LAR } \\
\end{array}$ & 0,11 & $\begin{array}{l}5,00 \mathrm{E}-08 \\
3,16 \mathrm{E}-07\end{array}$ & 15,84 & $\begin{array}{r}2,26 \mathrm{E}-08 \\
1,94 \mathrm{E}-07\end{array}$ & 11,64 & $\begin{array}{l}\text { 1,03E-08 } \\
\mathbf{8 , 8 5 E - 0 8}\end{array}$ & 11,64 \\
\hline
\end{tabular}

*Радионуклиды плутония: ${ }^{238} \mathrm{Pu},{ }^{239} \mathrm{Pu},{ }^{240} \mathrm{Pu},{ }^{241} \mathrm{Pu},{ }^{242} \mathrm{Pu}$.

Таблица 22

Пожизненные атрибутивные риски (LAR) онкозаболеваемости от суммарного набора радионуклидов и отдельных радионуклидов от выбросов БРЕСТ-ОД-300 (возраст при поступлении радионуклидов: 5 лет, 20 лет, 50 лет; женщины)

\begin{tabular}{|c|c|c|c|c|c|c|c|}
\hline $\begin{array}{l}\text { Радио- } \\
\text { нуклид }\end{array}$ & $\begin{array}{c}\text { Годовая } \\
\text { эфффективная } \\
\text { доза (мкЗв) }\end{array}$ & $\begin{array}{l}\text { LAR } \\
\text { (5 лет) }\end{array}$ & \begin{tabular}{|c|} 
Вклад радио- \\
нуклида в LAR \\
(5 лет), \%
\end{tabular} & $\begin{array}{c}\text { LAR } \\
\text { (20 лет) }\end{array}$ & \begin{tabular}{|c|} 
Вклад радио- \\
нуклида в LAR \\
(20 лет), \%
\end{tabular} & $\begin{array}{c}\text { LAR } \\
\text { (50 лет) }\end{array}$ & \begin{tabular}{|c|} 
Вклад радио- \\
нуклида в LAR \\
(50 лет), \%
\end{tabular} \\
\hline \multicolumn{8}{|c|}{ Ингаляционное поступление } \\
\hline $\begin{array}{l}\text { Радиоак- } \\
\text { тивные } \\
\text { благород- } \\
\text { ные газы }\end{array}$ & 0,11 & $2,02 E-08$ & 4,04 & $1,42 \mathrm{E}-08$ & 4,84 & $5,13 \mathrm{E}-09$ & 4,50 \\
\hline $\begin{array}{c}{ }^{14} \mathrm{C} \\
{ }^{3} \mathrm{H} \\
\mathrm{Pu}^{*} \\
{ }^{210} \mathrm{Po}\end{array}$ & $\begin{array}{l}1,22 \\
0,73 \\
0,49 \\
0,17\end{array}$ & $\begin{array}{l}2,32 \mathrm{E}-07 \\
1,40 \mathrm{E}-07 \\
1,73 \mathrm{E}-08 \\
5,50 \mathrm{E}-08\end{array}$ & $\begin{array}{c}46,46 \\
28,03 \\
3,46 \\
11,01\end{array}$ & $\begin{array}{l}1,35 \mathrm{E}-07 \\
8,09 \mathrm{E}-08 \\
9,79 \mathrm{E}-09 \\
3,46 \mathrm{E}-08\end{array}$ & $\begin{array}{c}46,01 \\
27,57 \\
3,34 \\
11,79\end{array}$ & $\begin{array}{l}5,47 \mathrm{E}-08 \\
3,17 \mathrm{E}-08 \\
3,23 \mathrm{E}-09 \\
1,01 \mathrm{E}-08\end{array}$ & $\begin{array}{c}48,02 \\
27,83 \\
2,84 \\
8,87\end{array}$ \\
\hline $\begin{array}{c}\text { Продукты } \\
\text { деления } \\
\boldsymbol{\Sigma} \text { LAR }\end{array}$ & 0,11 & $\begin{array}{l}3,49 \mathrm{E}-08 \\
4,99 \mathrm{E}-07\end{array}$ & 6,99 & $\begin{array}{l}1,89 \mathrm{E}-08 \\
2,94 \mathrm{E}-07\end{array}$ & 6,44 & $\begin{array}{l}9,06 \mathrm{E}-09 \\
\mathbf{1 , 1 4 E - 0 7}\end{array}$ & 7,95 \\
\hline
\end{tabular}

*Радионуклиды плутония: ${ }^{238} \mathrm{Pu},{ }^{239} \mathrm{Pu},{ }^{240} \mathrm{Pu},{ }^{241} \mathrm{Pu},{ }^{242} \mathrm{Pu}$. 


\section{Литература}

1. Радиационная защита и безопасность источников излучения: Международные основные нормы безопасности. Промежуточное издание. Общие требования безопасности. Серия норм МАГАТЭ по безопасности № GSR Part 3 (Interim). Вена: МАГАТЭ, 2011. 311 с.

2. Публикация 103 Международной комиссии по радиационной защите (МКРЗ): пер. с англ. /Под общей ред. М.Ф. Киселёва и Н.К. Шандалы. М.: Изд. ООО ПКФ «Алана», 2009. 312 с. [Электронный ресурс]. URL: http://www.icrp.org/docs/P103_Russian.pdf (дата обращения 20.10.2018).

3. Адамов Е.О., Джалавян А.В., Лопаткин А.В., Молоканов Н.А., Муравьёв Е.В., Орлов В.В., Калякин С.Г., Рачков В.И., Троянов В.М., Авронин Е.Н., Иванов В.Б., Алексахин Р.М. Концептуальные положения стратегии развития ядерной энергетики России в перспективе до 2100 г. //Атомная энергия. 2012. Т. 112, вып. 6. С. 319-330.

4. Адамов Е.О., Ганев И.Х. Экологически безупречная ядерная энергетика. М.: НИКИЭТ им. Н.А. Доллежаля, 2007. 145 с.

5. Иванов В.К., Чекин С.Ю., Меняйло А.Н., Максютов М.А., Туманов К.А., Кащеева П.В., Ловачёв C.C., Лопаткин А.В. Уровни радиологической защиты населения при реализации принципа радиационной эквивалентности: риск-ориентированный подход //Радиация и риск. 2018. Т. 27, № 3. С. 9-23.

6. Preston D.L., Shimizu Y., Pierce D.A., Suyama A., Mabuchi K. Studies of mortality of atomic bomb survivors. Report 13: Solid cancer and noncancer disease mortality: 1950-1997//Radiat. Res. 2003. V. 160, N 4. P. 381-407.

7. Preston D.L., Ron E., Tokuoka S., Funamoto S., Nishi N., Soda M., Mabuchi K., Kodama K. Solid cancer incidence in atomic bomb survivors: 1958-1998//Radiat. Res. 2007. V. 168, N 1. P. 1-64.

8. Иванов В.К. Радиологическая защита персонала: риск-ориентированный подход. М.: Общественный совет Госкорпорации «Росатом», 2018. 16 с.

9. Нормы радиационной безопасности (НРБ-99/2009). Санитарные правила и нормативы СанПиН 2.6.1.2523-09. М.: Федеральный центр гигиены и эпидемиологии Роспотребнадзора, 2009. 100 с.

10. Иванов В.К., Чекин С.Ю., Меняйло А.Н., Максютов М.А., Туманов К.А., Кащеева П.В., Ловачёв С.С., Адамов Е.О., Лопаткин А.В. Сравнительный анализ уровней «радиотоксичности» отдельных радионуклидов ОЯТ реакторов БРЕСТ и ВВЭР при различных временах выдержки на основе современных моделей «доза-эффект» МКРЗ //Радиация и риск. 2018. Т. 27, № 4. С. 8-27.

11. Меняйло А.Н., Чекин С.Ю., Кащеев В.В., Максютов М.А., Корело А.М., Туманов К.А., Пряхин Е.А., Ловачёв С.С., Карпенко С.В., Кащеева П.В., Иванов В.К. Пожизненный радиационный риск в результате внешнего и внутреннего облучения: метод оценки //Радиация и риск. 2018. Т. 27, № 1. С. 8-21.

12. Спирин Е.В., Алексахин Р.М., Бажанов А.А. Структура дозы облучения населения при эксплуатации предприятий опытного демонстрационного энергокомплекса //Атомная энергия. 2018. Т. 124, вып. 3. С. 169-173.

13. Крышев И.И., Сазыкина Т.Г., Крышев А.И., Дмитриева М.А., Лунёва К.В., Газиев И.Я., Пахомов А.Ю., Пахомова И.А. Оценка радиоэкологического воздействия объектов использования атомной энергии по данным радиационного мониторинга окружающей среды //Ядерная и радиационная безопасность. Выпуск 12. М.: Госкорпорация «Росатом», 2012. С. 139-160.

14. Kesminiene A., Evrard A-S., Ivanov V.K., Malakhova I.V., Kurtinaitise J., Stengrevics A., Tekkel M., Chekin S., Drozdovitch V., Gavrilin Y., Golovanov I., Kryuchkov V.P., Maceika E., Mirkhaidarov A.K., Polyakov S., Tenet V., Tukov A.R., Byrnes G., Cardis E. Risk of thyroid cancer among Chernobyl liquidators //Radiat. Res. 2012. V. 178. P. 425-436; DOI: 10.1667/RR2975.1.

15. Ivanov V.K., Tsyb A.F., Konogorov A.P., Rastopchin E.M., Khait S.E. Case-control analysis of leukaemia among Chernobyl accident emergency workers residing in the Russian Federation, 1986-1993 //J. Radiol. Prot. 1997. V. 17, N 3. P. 137-157. 
16. Konogorov A.P., Ivanov V.K., Chekin S.Yu., Khait S.E. A case-control analysis of leukemia in accident emergency workers of Chernobyl //J. Environ. Pathol. Toxicol. Oncol. 2000. V. 19, N 1-2. P. 143-151.

17. Ivanov V.K., Maksioutov M.A., Chekin S.Yu., Petrov A.V., Biryukov A.P., Kruglova Z.G., Matyash V.A., Tsyb A.F., Manton K.G., Kravchenko J.S. The risk of radiation-induced cerebrovascular disease in Chernobyl emergency workers //Health Phys. 2006. V. 90, N 3. P. 199-207.

\title{
Radiation risk safety limits for the public: operating Russian nuclear power plants; predictive estimates for the fast-neutron reactor BREST, operating under normal conditions
}

\author{
Ivanov V.K. ${ }^{1,2}$, Chekin S.Yu. ${ }^{1,2}$, Menyajlo A.N. ${ }^{1,2}$, Lovachev S.S. ${ }^{1,2}$, Korelo A.M. ${ }^{1,2}$ \\ ${ }^{1}$ A. Tsyb MRRC, Obninsk; \\ ${ }^{2}$ Medinfo LLC, Obninsk
}

\begin{abstract}
The IAEA publication on international basic safety standards highlights the necessity to establish dose limits for public, as well as limits of radiation risk of developing cancer. Results and conclusions of large-scale epidemiological studies, first of all the studies of health effects of atomic bombings of Hiroshima and Nagasaki in 1945 made the fulfillment of the above requirement possible. In the current Russian national safety standards (2009) there are three basic dose limits expressed as radiation risks for the population: $5 \cdot 10^{-5} \mathrm{y}^{-1}-$ in case of normal operation; $1 \cdot 10^{-5} \mathrm{y}^{-1}-$ sources of potential exposure to radiation; $1 \cdot 10^{-6} \mathrm{y}^{-1}-$ negligible risk. Levels of radiation safety for the population living near the nuclear facilities were estimated as lifetime attributable risk (LAR) values with the use of the models described in the ICRP 103 publication. The same method was used for making predictive assessment of safety levels of the fast neutrons reactor, BREST. LAR values for cancer development among the population living near the operating NPPs and predictive assessment of safety levels of the fast neutrons reactor, BREST, meet the current Russian national radiation safety standards - NRB-99/2009.
\end{abstract}

Key word: radiation dose; radiation risk; IAEA requirements; model "dose-effect»; epidemiological data of health effects of atomic bombings of Hiroshima and Nagasaki; NRB-99/2009 requirements; lifetime attributable risk; population living near NPP; BREST reactor; fast neutron reactor, negligible risk.

\section{References}

1. Radiation Protection and Safety of Radiation Sources. International Basic Safety Standards, GSR Part 3 (Interim), General Safety Requirements. Vienna, IAEA, 2011. 311 p. (In Russian).

2. ICRP, 2007. The 2007 Recommendations of the International Commission on Radiological Protection. ICRP Publication 103. Ann. ICRP 37 (2-4).

3. Adamov E.O., Dzhalavyan A.V., Lopatkin A.V., Molokanov N.A., Muravyov E.V., Orlov V.V., Kalyakin S.G., Rachkov V.I., Troyanov V.M., Avrorin E.N., Ivanov V.B., Aleksakhin R.M. Conceptual framework of a strategy for the development of nuclear power in Russia to 2100. Atomnaya energiya - Atomic Energy, 2012, vol. 112, no. 6, pp. 319-330. (In Russian).

4. Adamov E.O., Ganev I.Kh. Environmentally pure nuclear power. Moscow, N. Dollezhal PERDI, 2007. 145 p. (In Russian).

5. Ivanov V.K., Chekin S.Yu., Menyajlo A.N., Maksioutov M.A., Tumanov K.A., Kashcheeva P.V., Lovachev S.S., Adamov E.O., Lopatkin A.V. Application of the radiation equivalence principle to

Ivanov V.K.* - Deputy Director, Chairman of RSCRP, Corresponding Member of RAS; Chekin S.Yu. - Head of Lab.; Menyajlo A.N. - Lead. Researcher, C. Sc., Biol.; Lovachev S.S. - Research Assistant; Korelo A.M. - Senior Researcher. A. Tsyb MRRC, Medinfo.

${ }^{*}$ Contacts: 4 Korolev str., Obninsk, Kaluga region, Russia, 249036. Tel.: (484) 399-33-90; e-mail: nrer@obninsk.com. 
estimation of levels of radiological protection of the population: risk-oriented approach. Radiatsiya $i$ risk Radiation and Risk, 2018, vol. 27, no. 3, pp. 9-23. (In Russian).

6. Preston D.L., Shimizu Y., Pierce D.A., Suyama A., Mabuchi K. Studies of mortality of atomic bomb survivors. Report 13: Solid cancer and noncancer disease mortality: 1950-1997. Radiat. Res., 2003, vol. 160 , no. 4, pp. 381-407.

7. Preston D.L., Ron E., Tokuoka S., Funamoto S., Nishi N., Soda M., Mabuchi K., Kodama K. Solid cancer incidence in atomic bomb survivors: 1958-1998. Radiat. Res., 2007, vol. 168, no. 1, pp. 1-64.

8. Ivanov V.K. Staff radiological protection: a risk- oriented approach. Moscow, Public Council of ROSATOM, 2018. $16 \mathrm{p}$.

9. Radiation safety standards (RSS-99/2009). Sanitary-epidemiological rules and standards. SP2.6.1.252309. Moscow, Federal Center of Hygiene and Epidemiology of Rospotrebnadzor, 2009. 100 p. (In Russian).

10. Ivanov V.K., Chekin S.Yu., Menyajlo A.N., Maksioutov M.A., Tumanov K.A., Kashcheeva P.V., Lovachev S.S., Adamov E.O., Lopatkin A.V. Comparative analysis of the levels of "radiotoxicity" of some radionuclides of SNF from BREST and VVER reactors at different decay time based on modern ICRP "doseeffect" models. Radiatsiya i risk - Radiation and Risk, 2018, vol. 27, no. 4, pp. 8-27. (In Russian).

11. Menyajlo A.N., Chekin S.Yu., Kashcheev V.V., Maksioutov M.A., Korelo A.M., Tumanov K.A., Pryakhin E.A., Lovachev S.S., Karpenko S.V., Kashcheeva P.V., Ivanov V.K. Lifetime attributable risks from external and internal exposure to radiation: method for estimating. Radiatsiya i risk - Radiation and Risk, 2018, vol. 27, no. 1, pp. 8-21. (In Russian).

12. Spirin E.V., Alexakhin R.M., Bazhanov A.A. The structure of the dose exposure for the population at exploitation of experimental demonstration power complex. Atomnaya energiya - Atomic Energy, 2018, vol. 124, no. 3, pp. 169-173. (In Russian).

13. Kryshev I.I., Sazykina T.G., Kryshev A.I., Dmitriyeva M.A., Luneva K.V., Gaziyev I.YA., Pakhomov A.Yu., Pakhomova I.A. Assessment of the radioecological impact of nuclear facilities using radiation monitoring of the environment. Yadernaya i radiatsionnaya bezopasnost - Nuclear and Radiation Safety, vol. 12. Moscow, Rosatom State Corporation, 2012, pp. 139-160.

14. Kesminiene A., Evrard A-S., Ivanov V.K., Malakhova I.V., Kurtinaitise J., Stengrevics A., Tekkel M., Chekin S., Drozdovitch V., Gavrilin Y., Golovanov I., Kryuchkov V.P., Maceika E., Mirkhaidarov A.K., Polyakov S., Tenet V., Tukov A.R., Byrnes G., Cardis E. Risk of thyroid cancer among Chernobyl liquidators. Radiat. Res., 2012, vol. 178, pp. 425-436. DOI: 10.1667/RR2975.1.

15. Ivanov V.K., Tsyb A.F., Konogorov A.P., Rastopchin E.M., Khait S.E. Case-control analysis of leukaemia among Chernobyl accident emergency workers residing in the Russian Federation, 1986-1993. J. Radiol. Prot., 1997, vol. 17, no. 3, pp. 137-157.

16. Konogorov A.P., Ivanov V.K., Chekin S.Yu., Khait S.E. A case-control analysis of leukemia in accident emergency workers of Chernobyl. J. Environ. Pathol. Toxicol. Oncol., 2000, vol. 19, no. 1-2, pp. 143-151.

17. Ivanov V.K., Maksioutov M.A., Chekin S.Yu., Petrov A.V., Biryukov A.P., Kruglova Z.G., Matyash V.A., Tsyb A.F., Manton K.G., Kravchenko J.S. The risk of radiation-induced cerebrovascular disease in Chernobyl emergency workers. Health Phys., 2006, vol. 90, no. 3, pp. 199-207. 